\title{
TOPOLOGY OF OPEN NONPOSITIVELY CURVED MANIFOLDS
}

\author{
IGOR BELEGRADEK
}

\begin{abstract}
This is a survey of topological properties of open, complete nonpositively curved manifolds which may have infinite volume. Topics include topology of ends, restrictions on the fundamental group, as well as a review of known examples.
\end{abstract}

Among many monographs and surveys on aspects of nonpositive curvature, none deals with topology of open complete nonpositively curved manifolds, and this paper aims to fill the void. Most of the material discussed here is not widely-known. A number of questions is posed, ranging from naive to hopelessly difficult. Proofs are supplied when there is no explicit reference, and as always, the author is solely responsible for mistakes.

This survey has a narrow focus and does not discuss topological properties of

- hyperbolic 3-manifolds [Thu, Kap01, CM04,

- open negatively pinched manifolds [Bel01, BK06,

- non-Riemannian nonpositively curved manifolds Dav08,

- compact nonpositively curved manifolds FJO07, Lüc10,

- higher rank locally symmetric spaces [Mar91, Ebe96, BF02a, Gel11] and their compactifications BJ05,

which are covered in the above references. We choose to work in the Riemannian setting which leads to some simplifications, even though many results hold in a far greater generality, and the references usually point to the strongest results available. Special attention is given to rank one manifolds, such as manifolds of negative curvature. Nonpositively curved manifolds are aspherical so we focus on groups of finite cohomological dimension (i.e. fundamental groups of aspherical manifolds), or better yet, groups of type $F$ (i.e. the fundamental groups of compact aspherical manifolds with boundary).

Conventions: unless stated otherwise, manifolds are smooth, metrics are Riemannian, and sectional curvature is denoted by $K$.

2010 Mathematics Subject classification. Primary 53C20. Keywords: nonpositive curvature, discrete group, open manifold, ends, negative curvature, finite volume, rank one. 
Acknowledgments: The author is grateful for NSF support (DMS-1105045). Thanks are due to Jim Davis, Denis Osin, Yunhui Wu, and the referee for correcting misstatements in the earlier version.

\section{Contents}

1. Flavors of negative curvature

2

2. Manifolds of dimensions two and three

5

3. Towards a rough classification of discrete isometry groups 8

4. Groups of non-parabolic isometries 9

5. Groups with rank one elements: prelude 11

6. Acylindrically hyperbolic groups and rank one elements 12

7. Bounded cohomology and rank one elements 15

8. Monod-Shalom's class and rank one elements 15

9. Groups that fix a point at infinity: prelude 17

10. Groups whose center contains a parabolic 19

11. Anchored groups and fixed points at infinity 20

12. Homotopy obstructions (after Gromov and Izeki-Nayatani) 23

13. Homeomorphism obstructions: exploiting $\mathbb{R}$ factors 25

14. Benefits of a lower curvature bound 27

15. Injectivity radius going to zero at infinity 28

16. Negatively curved manifolds with uniform volume bound 30

17. Non-aspherical ends of nonpositively curved manifolds 31

18. Riemannian hyperbolization (after Ontaneda) 34

19. Topology of known manifolds of bounded negative curvature 37

20. Zoo of finite volume rank one manifolds 40

References 43

\section{Flavors of negative CuRvature}

A Hadamard manifold is a connected simply-connected complete manifold of $K \leq 0$. By the Cartan-Hadamard theorem, any Hadamard manifold is diffeomorphic to a Euclidean space. Thus any complete manifold of $K \leq 0$ is the quotient of a Hadamard manifold by a discrete torsion-free isometry 
group (torsion-freeness can be seen geometrically: any finite isometry group of a Hadamard manifold fixes the circumcenter of its orbit, or topologically: a nontrivial finite group has infinite cohomological dimension so it cannot act freely on a contractible manifold).

A Hadamard manifold is a visibility manifold if any two points at infinity can be joined by a geodesic. A flat in $X$ is a convex subset isometric to a Euclidean space. A flat half plane in $X$ is a convex subset isometric to a Euclidean half plane. An infinitesimal flat of a geodesic is the space of parallel Jacobi fields along a geodesic. A geodesic is called rank one if its infinitesimal flat is one-dimensional. A complete manifold of $K \leq 0$ has rank one if it contain a complete (i.e. defined for all times) geodesic of rank one.

The following conditions on a Hadamard manifold $X$ represent various manifestations of negative curvature:

(1) $K$ is bounded above by a negative constant,

(2) $X$ is Gromov hyperbolic,

(3) $X$ is a visibility manifold,

(4) $K<0$,

(5) $X$ contains no flat half plane,

(6) $X$ contains a complete geodesic that does not bound a flat half plane.

The implications $(1) \Rightarrow(4) \Rightarrow(5) \Rightarrow(6)$ and $(1) \Rightarrow(2) \Rightarrow(3) \Rightarrow(5) \Rightarrow(6)$ are immediate from definitions except for $(2) \Rightarrow(3)$ which can be found in BH99, Lemma VIII.3.2].

Proposition 1.1. Every other implication fails in dimension two.

Remark 1.2. There are of course analogs of (5) or (6) such as "no flat strip" or "no flat plane", see also various axioms on [EO73], but the above list is what comes up most often.

Remark 1.3. Kleiner Kle99 proved that a Hadamard manifold has rank one if and only if it contains a complete geodesic that does not lie in a two-dimensional flat (see [Bal95, Proposition IV.4.4] for the case when Iso $(X)$ satisfies the duality condition, e.g. contains a lattice). Since (6) is intermediate between the two conditions, it is equivalent to them.

Proof of Proposition 1.1. That $(2) \nRightarrow(4)$ follows by doubling along the boundary any nonpositively curved compact surface of negative Euler characteristic whose metric is cylindrical near the boundary; the universal cover is hyperbolic because hyperbolicity is a quasiisometry invariant, but it contains a flat strip.

To show that $(3) \nRightarrow(2)$ recall a consequence of the Gauss-Bonnet theorem, mentioned on [BGS85, page 57], that a 2-dimensional Hadamard manifold $X$ is visibility if and only if for some $p \in X$ the total curvature of every sector 
bounded by two rays that start at $p$ is infinite. Any smooth nonpositive function $K$ on $[0, \infty)$ can be realized as the curvature of a rotationally symmetric metric $d r^{2}+f(r)^{2} d \theta$ on $\mathbb{R}^{2}$, namely, $f$ is a unique solution of $f^{\prime \prime}+K f=0, f(0)=1$, $f^{\prime}(0)=1$. Note that $f(r) \geq r$ by Sturm comparison for ordinary differential equations, and the total curvature equals $2 \pi \int_{0}^{\infty} K f d r$. Let $I_{i}$ be a sequence of disjoint compact subintervals of $(0, \infty)$ such that $I_{i}$ has length $i$, and let $K$ be a smooth negative function on $[0, \infty)$ that equals $-\frac{1}{i}$ on $I_{i}$. The associated rotationally symmetric metric has infinite total curvature on every sector from the origin, so it is visibility, and it contains arbitrary large regions of curvature $-\frac{1}{i}$, which contain triangles violating $\delta$-hyperbolicity for any $\delta$.

That $(4) \nRightarrow(3)$ is also shown in [EO73, Example 5.10]: modifying the argument of the previous paragraph yields a rotationally symmetric metric with $K<0$ and finite total curvature.

To see that $(6) \nRightarrow(5)$ start from a finite volume complete hyperbolic metric on a punctured torus, and modify the metric near the end to a complete metric of $K \leq 0$ that is a cylinder outside a compact set. The cylinder lifts to a flat half plane so (5) fails, but any geodesic through a point of $K<0$ satisfies (6).

The other non-implications are formal consequence of the above implications and non-implications.

Remark 1.4. The non-implications in the above proof are justified by two dimensional examples, and it seems that similar examples in higher dimensions can be produced via iterated warping with $\cosh r$, i.e. replacing $X$ with $\mathbb{R} \times \cosh r X$, which contains $X$ as a totally geodesic submanifold. For some non-implications we need to insert the warped product as a convex subset in a closed manifold of $K \leq 0$ following Ontaneda, see [NP13]. The warping clearly preserves conditions (1), (4), (5), (6).

Question 1.5. Does the warping with $\cosh r$ preserve (2) and (3)?

One wants to understand the relations among (1)-(6) when Iso $(X)$ is large.

Example 1.6. If Iso $(X)$ is cocompact, then clearly $(1) \Leftrightarrow(4)$, and also $(2) \Leftrightarrow$ $(3) \Leftrightarrow(5)$; in fact, Gromov hyperbolicity is equivalent to uniform visibility for proper CAT(0) spaces [BH99, Proposition III.3.1], and visibility is equivalent to the non-existence of a 2-flat for proper CAT( 0$)$ spaces with cocompact isometry groups BH99, Theorem II.9.33]. For 2-dimensional Hadamard manifolds with cocompact isometry group $(5) \Leftrightarrow(6)$ because if $X$ contains a flat half plane and has cocompact isometry group, then $X$ is isometric to $\mathbb{R}^{2}$. On the other hand, $(6) \nRightarrow(5)$ in dimensions $>2$ with examples given by the Heintze manifolds, obtained by chopping of cusps of a finite volume complete hyperbolic manifold, changing the metric near the cusp a metric of $K \leq 0$ with totally geodesic 
flat boundary, and doubling along the boundary; the boundary lifts to a flat of dimension $>1$, while any geodesic through a point of $K<0$ has rank one.

Example 1.7. If $\operatorname{dim}(X)=2$ and $\operatorname{Iso}(X)$ contains a lattice, then either $X$ is isometric to $\mathbb{R}^{2}$ or $X$ is visibility [Ebe79, Proposition 2.5] so that (6) $\Leftrightarrow(3)$.

Example 1.8. If $\operatorname{dim}(X)>2$, then $(6) \nRightarrow(3)$; indeed Nguyen Phan $[\mathrm{NPb}$ and $\mathrm{Wu} \mathrm{Wu}$ show that the universal covers of the finite volume manifolds of $K<0$ constructed in Fuj88 are not visibility, so $(4) \nRightarrow(3)$.

Example 1.9. Eberlein showed [Ebe80] that the ends of a finite volume complete manifold with bounded nonpositive curvature and visibility universal cover are $\pi_{1}$-injectively embedded. This is not the case for Buyalo's example Buy93 of a finite volume complete 4 -manifold of $-1<K<0$; thus its universal cover is not visibility.

Remark 1.10. Let us compare (3) and (4) in higher dimensions. Non-visibility of $X$ can be checked by studying its two-dimensional totally geodesic submanifolds, cf. $[\mathrm{NPb}, \mathrm{Wu}$, while proving visibility of $X$ gets much harder, and indeed, it is a strong restriction on $X$ with a variety of consequences for the geometry of horoballs, Tits boundary, and isometry groups. By contrast, the condition " $K<0$ " is easy to verify but has few implications. Either condition implies that every complete geodesic in $X$ has rank one.

\section{MANifolds of Dimensions TWO AND THREE}

A classification of open connected 2-manifolds goes back to Kerékjártó Ker23; see [Ric63, Gol71, Sie] for more recent accounts.

Richards [Ric63, Theorem 3] proved that every open surface is obtained from $S^{2}$ by removing a closed totally disconnected subset, and then removing a finite or countable family of disjoint closed disks and attaching handles or Möbius bands along boundaries of the disks.

Remark 2.1. Any closed totally disconnected subset $T \subset S^{2}$ can be moved by an ambient homeomorphism to a subset of the standard Cantor set. (Like any compact totally disconnected metric space, $T$ is homeomorphic to a subset $Q$ of the Cantor set in $S^{2}$. The homeomorphism type of a planar surface is determined by the homeomorphism type of its the space of ends Ric63, Theorem 1], which for $S^{2} \backslash T, S^{2} \backslash Q$ are identified with $T, Q$, respectively. Hence there is a homeomorphism $S^{2} \backslash T \rightarrow S^{2} \backslash Q$, which extends to a homeomorphism of the end compactifications $S^{2} \rightarrow S^{2}$ mapping $T$ to $Q$ ).

The uniformization theorem equips any connected surface with a constant curvature metric, which can be chosen hyperbolic for open surfaces: 
Theorem 2.2. Any open connected 2-manifold admits a complete metric of constant negative curvature.

Proof. Given an open manifold $M$, we equip it with a complete Riemannian metric, and pull the metric back to the universal cover $\widetilde{M}$, where $\pi_{1}(M)$ acts isometrically, and hence by preserving the conformal class of the metric. By the uniformization theorem $\widetilde{M}$ is conformal to the hyperbolic plane $\mathbf{H}^{2}$ or to $\mathbb{C}$. A self-diffeomorphism of $\mathbf{H}^{2}$ that preserve the conformal class of the hyperbolic metric is an isometry of $\mathbf{H}^{2}$. A self-diffeomorphism of $\mathbb{C}$ that preserves the conformal class of the Euclidean metric is of the form $z \rightarrow a z+b$ or $z \rightarrow a \bar{z}+b$, and hence it either is an isometry (i.e. $a \bar{a}=1$ ), or its square has a fixed point (in fact, $z \rightarrow a z+b$ fixes $\frac{b}{1-a}$ and similarly for the square of $z \rightarrow a \bar{z}+b$ ). The deck-transformation $\pi_{1}(M)$-action is free, so it preserves the hyperbolic or the Euclidean metric. Thus $M$ admits a complete metric of constant curvature -1 or 0 . Finally, a flat open 2-manifold is $\mathbb{R}^{2}$, an annulus, or a Möbius band, so it also admits a complete hyperbolic metric.

Most of what is known on the geometrization of an open 3-manifold requires it to have finitely generated fundamental group, or better yet to be the interior of a compact manifold.

Theorem 2.3. The interior of any compact aspherical 3-manifold with nonempty boundary admits a complete metric of $-1 \leq K \leq 0$.

Proof. We refer to Kap01 for the terminology used in this proof. Let $N$ be a compact aspherical 3-manifold. Asphericity implies that $N$ contains no essential 2-sphere or projective plane. There is a decomposition of $N$ along incompressible 2-sided tori and Klein bottles into Seifert and atoroidal pieces, see [BS87a, which extends previous proofs by Johannson and Jaco-Shalen to the non-orientable case. Each piece is $\pi_{1}$-injectively embedded and hence aspherical.

Atoroidal pieces contain no essential annuli or Möbius bands whose boundary circles lie on the components of $\partial N$ of zero Euler characteristic (for the orientable case see e.g. [Hat, Lemma 1.16], and the non-orientable case follows by passing to the orientation cover for an essential annulus or Möbius band stays essential in the orientation cover, and a virtually Seifert piece is Seifert).

Define the pared manifold structure on every atoroidal piece $\mathrm{N}$ by letting its parabolic locus $P$ be the union of boundary components of zero Euler characteristic. Thurston's hyperbolization theorem [Kap01, Theorem 1.43] gives $(N, P)$ a geometrically finite hyperbolic structure whose parabolic subgroups are of rank 2 and bijectively corresponding to components of $P$. Every Seifert piece has a nonpositively curved metric with totally geodesic (flat) boundary by 
Leeb Lee95. In the same paper Leeb gives a gluing procedure for identifying rank 2 cusps and flat boundary components of Seifert pieces. (Leeb assumes that hyperbolic pieces have boundary of zero Euler characteristic but this does not matter since his gluing runs in the cusps, which in our case have rank 2). The result is a complete nonpositively curved metric on the interior of $N$.

Remark 2.4. A variation of the above proof identifies $N$ with a compact locally convex $C^{1}$ manifold of $K \leq 0$ with boundary, namely, replace geometrically finite pieces with the $\varepsilon$-neighborhoods of their convex cores, where the cusps are chopped off, and the metric at cusps are modified so that their boundaries are flat and totally geodesic. Thus the group $\pi_{1}(N)$ is $\operatorname{CAT}(0)$, which was first noted in [Bri01, Theorem 4.3] with a slightly different proof.

Remark 2.5. More information on 3-manifold groups can be found in the survey [AFW]; in particular, if $M$ is open aspherical 3-manifold with finitely generated fundamental group, then the advances on the virtual Haken conjecture imply that $\pi_{1}(M)$ virtually embeds into a right angled Artin group, which has a number of group-theoretic consequences, e.g. $\pi_{1}(M)$ is linear over $\mathbb{Z}$.

By Scott/Shalen compact core theorem any open aspherical 3-manifold $M$ with finitely generated deformation retracts to a compact codimension zero submanifold (to which Theorem 2.3 applies) but the topology of $M$ is still a mystery.

A open 3-manifold is called tame if it is homeomorphic to the interior of a compact manifold. Marden's Tameness Conjecture predicted tameness of every open complete 3 -manifold with $K \equiv-1$ and finitely generated fundamental group, and it was proved by Agol Ago and Gabai-Calegari CG06. The following question is still open:

Question 2.6. Let $M$ be an open 3-manifold with a complete metric of $K \leq 0$ and finitely generated fundamental group. Is $M$ tame?

The same question is open for manifolds of $K \leq-1$ or $-1 \leq K \leq 0$. The answer is yes under any of the following assumptions:

- $M$ admits a complete negatively pinched metric (due to Bowditch Bow10 who built on proofs of Marden's Tameness Conjecture by Agol, CalegariGabai, and Soma).

- $M$ is a cover of the interior of a compact manifold (as proved by LongReid in Can08 that combines results of Simon with the proof of Tameness Conjecture; here the assumption that $M$ has $K \leq 0$ is not needed).

- $M$ has a complete metric of $-1 \leq K \leq 0$ and Inj Rad $\rightarrow 0$ (as proved by Schroeder [BGS85, Appendix 2]). 
By contrast, there exits non-tame, open 3-manifolds with universal cover diffeomorphic to $\mathbb{R}^{3}$ and fundamental groups isomorphic to $\mathbb{Z}$ [ST89] and $\mathbb{Z} * \mathbb{Z}$ FG07.

Example 2.7. The non-tame fake open solid torus in [ST89] does not admit a complete metric of $K \leq 0$ because $\mathbb{R}^{2}$-bundles over $S^{1}$ are the only open, complete 3 -manifolds of $K \leq 0$ with infinite cyclic fundamental group. (The isometric $\mathbb{Z}$-action in the universal cover must stabilize a geodesic or a horoball, in the former case the nearest point projection to the geodesic descends to an $\mathbb{R}^{2}$-bundle over $S^{1}$, while in the latter case the quotient is the product of $\mathbb{R}$ and an open surface homotopy equivalent to a circle, which is an $\mathbb{R}$-bundle over $\left.S^{1}\right)$.

\section{TOWARDS A ROUGH CLASSIFICATION OF DISCRETE ISOMETRY GROUPS}

In this section we sketch a higher-dimensional analog of the classification of open complete constant curvature surfaces; details appear in Sections 411 . We refer to [BGS85, Ebe96, BH99] and Section 1 for background on Hadamard manifolds, adopt the following notations:

- $X$ is a Hadamard manifold with ideal boundary $X(\infty)$,

- Iso $(X)$ is the isometry group of $X$,

- $\Gamma$ is a subgroup of $\operatorname{Iso}(X)$,

and consider the following three classes of complete manifolds of $K \leq 0$ of the form $X / G$ where $\Gamma \leq \operatorname{Iso}(X)$ is discrete and torsion-free:

(1) $\Gamma$ contains no parabolic elements.

(2) $\Gamma$ contains a rank one element.

(3) $\Gamma$ fixes a point $\xi \in X(\infty)$, and the associated $\Gamma$-action on the space $L_{\xi}$ of lines asymptotic to $\xi$ is free and properly discontinuous.

There are severe algebraic restrictions on $\Gamma$ in the cases (1)-(2), and in the case (3) the manifold $X / \Gamma$ is diffeomorphic to the product of $\mathbb{R}$ and $L_{\xi} / \Gamma$, where $L_{\xi}$ is diffeomorphic to a Euclidean space.

Example 3.1. $\Gamma$ satisfies (3) if it stabilizes a horoball. (Indeed, $L_{\xi}$ is equivariantly diffeomorphic to a horosphere, and the discreteness of $\Gamma$ implies that its action on $L_{\xi}$ is properly discontinuous).

Question 3.2. Suppose $X$ has rank one. Does every discrete torsion-free isometry group $\Gamma$ of $X$ satisfy one of the conditions (1), (2), (3) above?

The answer is yes if $X$ is visibility (i.e. any two points of $X(\infty)$ are endpoints of a geodesic, which happens e.g. if $K \leq-1$ ), see Corollary 9.8 . 
Without any assumption on $X$ the answer is no, e.g. when $X / \Gamma$ is the product of two finite volume, complete, open, hyperbolic surfaces.

Remark 3.3. (a) The classes (1), (2), (3) are clearly not disjoint, e.g. a the cyclic group generated by a translation in $\mathbb{R}^{n}$ lies in (1) and (3). On the other hand, any (discrete) subgroup satisfying (2) and (3) is virtually cyclic, see Proposition 8.3 .

(b) A given group may be isomorphic to three different isometry groups satisfying (1), (2), (3) respectively.

(c) If $\Gamma \leq \operatorname{Iso}(X)$ is a discrete subgroup, then the requirement " $\Gamma$ is isomorphic to a discrete isometry group of a Hadamard manifold that satisfies (3)" does not restrict $\Gamma$ because the isometric $\Gamma$-action on the warped product Hadamard manifold $\mathbb{R} \times e_{e^{r}} X$ stabilizes a horoball. On the other hand, if we fix the dimension, this does become a nontrivial restriction as follows.

(d) Define the Euclidean action dimension as the smallest dimension of a Euclidean space on which $\Gamma$ acts smoothly and properly discontinuously, and if the Euclidean action dimension of $\Gamma$ equals $\operatorname{dim}(X)$, then $\Gamma$-action on $X$ cannot satisfy (3) because $L_{\xi}$ is diffeomorphic to the Euclidean space of lower dimension. See [BKK02, BF02a, Yoo04, Des06] for computations of a related invariant called the action dimension which usually equals the Euclidean action dimension.

\section{Groups of NON-PARABolic isometries}

In this section we discuss groups in the class (1) of Section 3. An isometry of $X$ is elliptic, axial, or parabolic if the minimum of its displacement function is zero, positive, or not attained, respectively. If $\gamma$ is a non-parabolic isometry, then the set $\operatorname{Min}(\gamma)$ of points where the displacement functions attains a minimum splits as $C_{\gamma} \times \mathbb{R}^{k}$, where $C_{\gamma}$ is a closed convex subset with $\gamma$ acting as the product of the trivial action on $C_{\gamma}$ and a translation on $\mathbb{R}^{k}$ BH99, Theorem II.7.1]. If $\gamma$ is axial, its axes are precisely the lines $\{x\} \times \mathbb{R}, x \in C_{\gamma}$.

Theorems 4.1, 4.3, 4.4(2ab) are part of the flat torus theorem "package" discovered by Gromoll-Wolf [GW71] and Lawson-Yau [LY72], and generalized in [BH99].

Theorem 4.1. Let $A \leq I s o(X)$ be an abelian discrete subgroup that consists of non-parabolic isometries. Set $\operatorname{Min}(A):=\cap_{a \in A} \operatorname{Min}(a)$. Then

(1) $\operatorname{Min}(A)$ is a nonempty, closed, convex $A$-invariant subset that splits as $C_{A} \times \mathbb{R}^{m}$ where $A$ acts trivially on $C_{A}$ and by translations on $\mathbb{R}^{m}$;

(2) A is finitely generated of rank $\leq m \leq \operatorname{dim}(X)$;

(3) A has finite intersection with each conjugacy class in $\operatorname{Iso}(X)$. 
Remark 4.2. Any periodic abelian discrete subgroup of $\operatorname{Iso}(X)$ is finite (because it is countable, hence locally finite, and so is a union of finite subgroups whose fixes points set is a descending family of totally geodesics submanifolds of $X$, which has to stabilize by dimension reasons). Thus abelian discrete groups of non-parabolic isometries are finitely generated, which is a key feature of the Riemannian setting. By contrast $\mathbb{Q}$ can act properly by non-parabolic isometries on a proper $\mathrm{CAT}(0)$ space, which is the product of a simplicial tree and a line [BH99, Example II.7.13].

Theorem 4.3. Let $A \leq I s o(X)$ be an abelian discrete subgroup that consists of axial isometries, and let $N \leq I s o(X)$ is a subgroup that normalizes $A$. Then

(4) $N$ stabilizes $\operatorname{Min}(A)$ and preserves its product decomposition;

(5) A is centralized by a finite index subgroup of $N$;

(6) $A$ is a virtual direct factor of $N$ if $N$ is finitely generated and $A \leq N$.

Proof of Theorems 4.1. 4.3. (1) is proved in [BGS85, Lemma 7.1(1)]. Discreteness of $A$ and (1) implies that $A$-action on $\mathbb{R}^{m}$ is properly discontinuous, so $A$ has rank $\leq m$, which gives (2). Claim (3) follows from BH99, Lemma II.7.17(2)], and (4), (5), (6) are parts of the flat torus theorem [BH99, Theorem II.7.1].

Theorem 4.4. Let $\Gamma \leq \operatorname{Iso}(X)$ be a subgroup without parabolic elements.

(1) If $H$ is commensurable to $\Gamma$, then $H$ is isomorphic to a discrete group of non-parabolic isometries of some Hadamard manifold.

(2) The following groups do not embed into $\Gamma$ :

(a) any solvable group that is not virtually abelian;

(b) the Baumslag-Solitar group $\left\langle x, y \mid x y^{m} x^{-1}=y^{l}\right\rangle$ with $m \neq \pm l$;

(c) $\pi_{1}(L)$, where $L$ is a closed aspherical 3-manifold that admits no metric of $K \leq 0$.

Proof. (1) is immediate via the induced representation construction [KL96, Theorem 2.3]. To prove (2a) note that $G$ must be polycyclic (combine finite generation of abelian discrete subgroups of non-parabolic isometries with Mal'cev's theorem that a solvable group whose abelian subgroups are finitely generated is polycyclic). Then proceed by induction on Hirsch length, and use Theorem 4.3 (6) to split virtual $\mathbb{Z}$-factors one at a time, see [BH99, Theorem II.7.16]. Also (2b) follows from Theorem 4.1(3), see [BH99, Theorem III.Г.1.1(iii)]. Finally, to prove (2c) invoke the solution of the virtual Haken conjecture Ago13; thus $L$ has a Haken finite cover, so it admits a metric of $K \leq 0$ by the main result in [KL96, Corollaries 2.6-2.7].

\section{Remark 4.5.}


(1) If a closed aspherical 3-manifold admits no metric of $K \leq 0$, then it is Seifert or graph (the manifold is virtually Haken Ago13 so the claim follows from [Lee95, KL96]).

(2) Closed aspherical Seifert manifolds that admit no metric of $K \leq 0$ are precisely those modelled on Nil, Sol, or $\widetilde{S L}_{2}(\mathbb{R})$ as easily follows from Theorems 4.1 (6), 4.4(2a), and the observation that the Seifert manifolds modelled on $\mathbb{R}^{3}$ or $\mathbb{H}^{2} \times \mathbb{R}$ are nonpositively curved.

(3) The problem which orientable closed graph manifolds admit metrics of $K \leq 0$ was resolved in BK95, BS04 who found several combinatorial criteria on the gluing data. (These papers only consider manifolds with no embedded Klein bottles, but the assumption can be easily removed as was explained to the author by Buyalo).

(4) A non-orientable closed 3-manifold admits a metric of $K \leq 0$ if and only if its orientation cover does [KL96].

(5) A closed aspherical graph manifold admits a metric of $K \leq 0$ if and only if its fundamental group virtually embeds into a right angled Artin group [Liu13].

(6) Kapovich-Leeb used Theorem 4.4(2c) to give other examples of groups that do not act on Hadamard spaces by non-parabolic isometries [KL96].

\section{Groups With RANK one ELEMENTS: PRELude}

Sections 58 collect what is known on the class (2) of Section 3 .

An axial isometry $\gamma$ has rank one if it has an axis that does not bound a flat half plane. This property can be characterized in terms of the splitting $\operatorname{Min}(\gamma) \cong C_{\gamma} \times \mathbb{R}^{k}$, namely, $\gamma$ has rank one if and only if $C_{\gamma}$ is compact.

Any discrete subgroup of Iso $(X)$ that normalizes a rank one element is virtually cyclic (in fact, the normalizer preserves the splitting, and hence fixes a point of $C_{\gamma}$ and stabilizing the corresponding axis).

Rank one isometries were introduced by Ballmann [Bal95, Theorem III.3.4], who proved that if $X$ is a rank one and $\Gamma \leq \operatorname{Iso}(X)$ is any subgroup satisfying the duality condition (e.g. a lattice), then $\Gamma$ contains a rank one element. He then used a ping pong argument to find a copy of non-cyclic free group inside $\Gamma$. Various aspects of isometry groups containing rank one elements were further studied in [BF02b, BB08, BF09, Ham09b, Ham12, CF10. In particular, the following is due to [BF09, Proposition 5.11] or [Ham09b, Theorem 1.1(4)]:

Theorem 5.1. If $\Gamma \leq \operatorname{Iso}(X)$ is a discrete subgroup that contains a rank one element and is not virtually- $\mathbb{Z}$, then $\Gamma$ contains a non-cyclic free subgroup consisting of rank one elements. 
Sisto proved [Sis, Theorem 1.4] that if $\Gamma$ in Theorem [5.1] is finitely generated, then its generic element has rank one, where "generic" roughly means that the probability that a word written in random finite generating set of $G$ represents a rank one element approaches 1 exponentially with the length of the word.

\section{ACYLINDRICALLY HYPERBOLIC GROUPS AND RANK ONE ELEMENTS}

In the last decade it was realized that many groups of geometric origin contain (suitably defined) rank one elements, which allowed for a uniform treatment of such groups and resulted in a host of applications. A crucial notion in these developments is acylindricity which goes back to Sela and Bowditch. In connection with rank one elements different versions of acylindricity were introduced and studied by Bestvina-Fujiwara BF02b, BF09, Hamenstädt Ham09a, DahmaniGuirardel-Osin [DGO], Sisto [Sis], and most recently Osin showed [Osi] that all these approaches are equivalent.

An isometric action of a group $G$ on a Gromov hyperbolic space $(X, d)$ is called

- non-elementary if its limit set consists of $>2$ points,

- acylindrical if for each $\varepsilon>0$ there are $R, N$ such that if $d(x, y) \geq R$, then at most $N$ elements $g \in G$ satisfy $d(x, g x) \leq \varepsilon$ and $d(y, g y) \leq \varepsilon$.

A group $G$ is acylindically hyperbolic if it admits a non-elementary acylindrical isometric action on a Gromov hyperbolic space.

The class of acylindically hyperbolic groups includes many groups of geometric origin, e.g. any subgroup of a relatively hyperbolic group that is not virtuallycyclic and does not lie is peripheral subgroup, or all but finitely many mapping class groups; see [Osi], for other examples.

Of particular importance for this section is the following result of Sisto Sis] who actually proves it for any group acting properly and isometrically on a proper CAT( 0$)$ space:

Theorem 6.1. (Sisto) If $\Gamma \leq I s o(X)$ is a discrete subgroup that contains a rank one element, then $\Gamma$ is virtually cyclic or acylindrically hyperbolic.

Dahmani-Guirardel-Osin DGO introduced a notion of a hyperbolically embedded subgroup of $G$, and Osin Osi proved that $G$ is acylindrically hyperbolic if and only if $G$ contains an infinite, proper, hyperbolically embedded subgroup. What Sisto actually showed is that any rank one element $\gamma \in \Gamma$ lies in a virtually cyclic hyperbolically embedded subgroup $E(\gamma)$. We omit the definition of a hyperbolically embedded subgroup, and just note that they are almost malnormal by [DGO, Proposition 4.33]:

Theorem 6.2. If $H$ is a hyperbolically embedded subgroup of a group $G$, then $H$ is almost malnormal in $G$, i.e. $H \cap g H^{-1}$ is finite for all $g \notin H$. 
Here are other applications [DGO, Osi], which hold in particular when $G$ is a discrete, non-virtually-cyclic subgroup of Iso $(X)$ containing a rank one element.

Theorem 6.3. If $G$ is acylindrically hyperbolic, then

(1) $G$ has a non-cyclic, normal, free subgroup,

(2) every countable group embeds into a quotient of $G$,

(3) every infinite subnormal subgroup of $G$ is acylindrically hyperbolic,

(4) $G$ has no nontrivial finite normal subgroups if and only if every conjugacy class in $G$ is infinite,

(5) every s-normal subgroup of $G$ is acylindrically hyperbolic,

(6) if $G$ equals the product of subgroups $G_{1}, \ldots, G_{k}$, then at least one $G_{i}$ is acylindrically hyperbolic.

(7) $G$ is not the direct product of infinite groups.

(8) any group commensurable to $G$ is acylindrically hyperbolic.

(9) any co-amenable subgroup of $G$ acylindrically hyperbolic.

Proof. Proofs of (1)-(4) are in DGO, Theorem 8.6, Lemma 8.11, Theorem 8.12] (1)-(2), while (5)-(7) are proved in Osi, Corollary 1.5, Proposition 1.7, Corollary 7.3], and (8) appears in [MO, Lemma 3.8].

The claim (9) is due to Osin who communicated to the author the following argument and kindly permitted to include it here. Suppose $G$ is acylindrically hyperbolic and $K \leq G$ is not. In the next paragraph we find a non-cyclic free subgroup $F \leq G$ with $F \cap K=\{1\}$. The group $F$ is not amenable, and its action by left translations on the set $G / H$ is free, so there is no $F$-invariant finitely additive probability measure on $G / K$. The contrapositive of (9) now follows because if $K$ were co-amenable, $G / K$ would admit a $G$-invariant (and hence $F$-invariant) finitely additive probability measure.

To construct $F$ note that Osi, Theorems 1.1-1.2] yield a non-elementary acylindrical $G$-action on a Gromov hyperbolic space for which the subgroup $K$ is either elliptic or else virtually- $\mathbb{Z}$ and contains a loxodromic element (we refer to Osi for terminology). If $K$ is elliptic, then Osi, Theorems 1.1] yields independent loxodromic elements $a, b \in G$. The standard ping-pong argument shows that for some $n \gg 1$, the subgroup $\left\langle a^{n}, b^{n}\right\rangle$ is free of rank 2 and all its non-trivial elements are loxodromic. In particular, $\left\langle a^{n}, b^{n}\right\rangle \cap K=\{1\}$. If $K$ is virtually- $\mathbb{Z}$ and contains a loxodromic element $c$, then [Osi, Theorems 1.1] gives loxodromic elements $a, b \in G$ such that $a, b, c$ are independent. Again by ping-pong $\left\langle a^{n}, b^{n}, c^{n}\right\rangle$ is free of rank 3 so that $\left\langle a^{n}, b^{n}\right\rangle \cap K=\{1\}$. Thus we get a non-cyclic free subgroup $F=\left\langle a^{n}, b^{n}\right\rangle$ with $F \cap K=\{1\}$, and in fact all nontrivial elements of $F$ are loxodromic.

A subgroup $K \leq G$ is subnormal if there are subgroups $G_{i} \leq G$ with $G_{0}=G$, $G_{k}=K$, and such that $G_{i}$ is a normal in $G_{i-1}$ for all $i=1, \ldots, k$. 
If a group $G$ equals the product of subgroups $G_{1}, \ldots, G_{k}$, one says that $G$ boundedly generated by $G_{1}, \ldots, G_{k}$.

Two groups are commensurable if they have isomorphic finite index subgroups.

A subgroup $K \leq G$ is s-normal if $K \cap g K g^{-1}$ is infinite for each $g \in G$. Thus the Baumslag-Solitar group $B(m, n)=\left\langle a, b \mid a b^{m}=b^{n} a\right\rangle$ is not acylindrically hyperbolic, except for $B(0,0)$, because $\langle b\rangle$ is s-normal and not acylindrically hyperbolic.

A subgroup $K \leq G$ is co-amenable if one of the following holds:

(1) every continuous affine $G$-action on a convex compact subset of a locally convex space with a $K$-fixed point has a $G$-fixed point;

(2) $\ell^{\infty}(G / K)$ has a $G$-invariant mean;

(3) $G / K$ has a $G$-invariant finitely additive probability measure;

(4) the inclusion $K \hookrightarrow G$ induces injections in bounded cohomology in all degrees with coefficients in any dual Banach $G$-module.

The equivalence $(1) \Leftrightarrow(2)$ is proved in Eym72, while $(2) \Leftrightarrow(3)$ follows from the standard correspondence between means and measures, and $(3) \Leftrightarrow(4)$ can be found in [MP03]. See also [Moo09] for leisurely discussion of co-amenability.

Here we are mainly interested in examples of non-amenable groups that admit co-amenable subgroups:

- A normal subgroup $N \unlhd G$ is co-amenable if and only if $G / N$ is amenable.

- If $K$ is co-amenable in $N$, and in turn $N$ is co-amenable in $G$, then $K$ is co-amenable in $G$.

- the image of a co-amenable subgroup under an epimorphism $G \rightarrow \bar{G}$ is co-amenable.

- If $\theta: K \rightarrow K$ is a monomorphism, and $G:=\left\langle K, t \mid t k t^{-1}=\theta(k), k \in K\right\rangle$ is the associated HNN-extension, then $K$ is co-amenable in $G$.

The first three facts above are straightforward, while the last one is due to Monod-Popa [MP03].

Example 6.4. Starting from a group $K$ that is not acylindrically hyperbolic one can use iterated HNN-extensions and extensions with amenable quotient to get many examples of non acylindrically hyperbolic groups. (These constructions preserve finiteness of cohomological dimension if the initial $K$ and every amenable quotient have finite cohomological dimension). 


\section{Bounded COHOMOLOGY AND RANK ONE ELEMENTS}

Bounded cohomology naturally appear in a variety of contexts, see e.g. Gro82, Mon06a. Of particular interest for our purposes is the comparison map

$$
\iota(G): H_{b}^{2}(G ; \mathbb{R}) \rightarrow H^{2}(G ; \mathbb{R})
$$

between the bounded and ordinary cohomology in degree two, which encodes some subtle group-theoretic properties:

- (Johnson) If $G$ is amenable, then $H^{p}(G ; \mathbb{R})=0$ for $p>0$ [Joh72, Nos90].

- (Burger-Monod) $\iota(G)$ is injective if $G$ is the fundamental group of an irreducible, finite volume complete manifold of $K \leq 0$, no local Euclidean de Rham factor, and rank $\geq 2$ [BM02].

- (Bavard) Injectivity of $\iota(G)$ is equivalent to vanishing of the stable commutator length on $[G, G]$ Bav91. Thus if $\iota(G)$ is non-injective, then there is $g \in[G, G]$ such that the minimal number of commutators needed to represent $g^{n}$ grows linearly with $n$.

Theorem 7.1. (Bestvina-Fujiwara, Osin) If $G$ is acylindrically hyperbolic, then the comparison map $\iota(\Gamma)$ has infinite dimensional kernel.

This was proved in [BF02b] for a class of groups which according to Osi] coincides with the class of acylindrically hyperbolic groups.

Remark 7.2. For discrete subgroup $\Gamma \leq \operatorname{Iso}(X)$ with rank one elements the above theorem was first established in [BF09]. Discreteness of $\Gamma$ in Theorem[7.1 can be weakened to the weak proper discontinuity [BF09, but it cannot be dropped, e.g. the projection of any irreducible lattice $\Lambda \leq \operatorname{Iso}\left(\mathbf{H}^{2}\right) \times \operatorname{Iso}\left(\mathbf{H}^{2}\right)$ to either factor acts on the hyperbolic plane isometrically, effectively, and by rank one isometries, but the comparison map $\iota(\Lambda)$ is injective BM02].

\section{Monod-Shalom's Class AND RANK ONE ELEMENTS}

In MS04, MS06, Monod-Shalom introduced and studied the following class of groups, which they thought of as a cohomological manifestation of negative curvature: Let $\mathcal{C}_{\text {reg }}$ be the class of countable groups $G$ such that $H_{b}^{2}\left(G ; \ell^{2}(G)\right) \neq 0$, which refers to the bounded cohomology of $G$ with coefficients in the regular representation.

A way to prove that $H_{b}^{2}\left(G ; \ell^{2}(G)\right) \neq 0$ is to show that the corresponding comparison map $H_{b}^{2}\left(G ; \ell^{2}(G)\right) \rightarrow H^{2}\left(G ; \ell^{2}(G)\right)$ has infinite dimensional kernel, which was done for many "hyperbolic-like" groups in [MMS04]. The following was proved by Hamenstädt [Ham08], and later from a different perspective by Hull-Osin [HO13]: 
Theorem 8.1. $\mathcal{C}_{\text {reg }}$ contains every countable acylindrically hyperbolic group, and hence any discrete subgroup $\Gamma \leq I$ so $(X)$ that contains a rank one element and is not virtually- $\mathbb{Z}$.

For discrete subgroup $\Gamma \leq \operatorname{Iso}(X)$ with rank one elements the above theorem was first established in Ham12].

As proved in [MS06, Chapter 7], examples of groups not in $\mathcal{C}_{\text {reg }}$ include

- amenable groups,

- products of at least two infinite groups,

- lattices in higher-rank simple Lie groups (over any local field),

- irreducible lattices in products of compactly generated non-amenable groups.

and the class $\mathcal{C}_{\text {reg }}$ is closed under

- passing to an infinite normal subgroup,

- passing to a co-amenable subgroup,

- measure equivalence.

We refer to [Fur11] for a survey on measure equivalence; e.g. commensurable groups are measure equivalent.

Question 8.2. Is every group in $\mathcal{C}_{\text {reg }}$ acylindrically hyperbolic?

To transition to our next topic, note that non-virtually-cyclic discrete groups with rank one elements never fix a point at infinity:

Proposition 8.3. If $\Gamma$ is discrete, fixes a point at infinity, and contains a rank one element, then $\Gamma$ is virtually cyclic.

Proof. This follows from [BF09, Section 6] provided $\Gamma$ satisfies the weak proper discontinuity condition, which is implied by discreteness. The idea is that if $g \in \Gamma$ has rank one, then either $\Gamma$ is virtually- $\mathbb{Z}$, or $\Gamma$ contains another rank one element $h$ such that their axis $A_{g}, A_{h}$ do not have the same sets of endpoints at infinity. A rank one element fixes precisely two points at infinity, the endpoints of its axis. Since $\Gamma$ has a fixed point, it must be a common endpoint of $A_{g}, A_{h}$, and this contradicts weak proper discontinuity: there is a subsegment $I$ of $A_{g}$ and an infinite subset $Q$ of $\Gamma$ such that the distances between the endpoints of $I$ and $g(I), g \in Q$ are uniformly bounded.

Example 8.4. The (non-discrete) stabilizer of a boundary point in the hyperbolic plane contains rank one elements without being virtually- $\mathbb{Z}$. 


\section{Groups that FiX A POINT AT Infinity: PRELUde}

Basic properties of horoballs, horospheres, and Busemann functions can be found in BGS85, Ebe96, Bal95. A horoball in $X$ is the Hausdorff limit of a sequence of metric balls in $X$ with radii going to infinity. A horosphere is the boundary of a horoball. Every point at infinity $\xi$ is represented by a Busemann function $b_{\xi}: X \rightarrow \mathbb{R}$, which is determined by $\xi$ up to an additive constant. The fibers of $b_{\xi}$ are the horospheres centered at $\xi$, and the sublevel sets of $b$ are horoballs centered at $\xi$. The function $b_{\xi}$ is a $C^{2}$ Riemannian submersion $X \rightarrow \mathbb{R}$, and in particular, each horosphere is diffeomorphic to the Euclidean space of dimension $\operatorname{dim}(X)-1$.

If $\Gamma$ fixes a point $\xi$ at infinity of $X$, then $\Gamma$ permutes horospheres centered at $\xi$, and associating to $\gamma \in \Gamma$ the distance by which it moves a horosphere to a concentric one defines a homomorphism $\Gamma \rightarrow \mathbb{R}$, which is in general nontrivial (think of the stabilizer of a point at infinity of the hyperbolic plane).

Let $L_{\xi}$ be the space of lines in $X$ asymptotic to $\xi$. The geodesic flow towards $\xi$ identifies $X$ with the total space of a principal $\mathbb{R}$-bundle over $L_{\xi}$, which is trivial as every horosphere centered at $\xi$ gives a section. In particular, $L_{\xi}$ has a structure of a smooth manifold diffeomorphic to a horosphere about $\xi$. If $\Gamma$ fixes $\xi$, then it acts smoothly on $L_{\xi}$.

Recall the condition (3) of Section 3. $\Gamma$ fixes a point $\xi \in X(\infty)$, and the associated $\Gamma$-action on the space $L_{\xi}$ of lines asymptotic to $\xi$ is free and properly discontinuous. Under this condition the principal $\mathbb{R}$-bundle $X \rightarrow L_{\xi}$ descends to an orientable (and hence trivial) real line bundle $X / \Gamma \rightarrow L_{\xi} / \Gamma$, so we get:

Lemma 9.1. If $\Gamma$ satisfies the condition (3) of Section 3, then $X / \Gamma$ is diffeomorphic to the product of $\mathbb{R}$ and $L_{\xi} / \Gamma$.

The prime example of a group satisfying (3) is a discrete torsion-free subgroup $\Gamma$ that stabilizes a horoball, in which case $\Gamma$ stabilizes every concentric horoball, so that $X$ is $\Gamma$-equivariantly is diffeomorphic to the product of $\mathbb{R}$ with a horosphere, and (3) follows because $\Gamma$ acts freely and properly discontinuously on $X$. More examples are needed:

Question 9.2. Let $\Gamma$ be any discrete torsion-free isometry group of $X$ whose fixed point set at infinity is nonempty.

- Does $\Gamma$ satisfies (3) for some $\xi$ ?

- If $\Gamma$ satisfies (3), does $\Gamma$ stabilize a horoball?

- What is the structure of $\Gamma$ if it does not stabilize a horoball?

Remark 9.3. (1) An axial isometry does not stabilize a horoball centered at an endpoint of one of its axis, but it can stabilize another horoball (e.g. translation in the plane stabilizes any half plane whose boundary is parallel to 
the translation axis).

(2) Any parabolic isometry stabilizes a horoball by Lemma 10.1 but different parabolics in $\Gamma$ can stabilize different horoballs.

(3) An elliptic element fixing a point at infinity stabilizes a horoball centered at the point (because it fixes a ray from a fixes point inside $X$ to the fixed point at infinity).

Question 9.4. Let $\Gamma \leq I s o(X)$ be discrete, containing a parabolic and no rank one elements. What conditions on $X$ ensure that $\Gamma$ fixes a point at infinity?

Recall that the limit set $\Lambda(\Gamma)$ is the set of accumulation points of the $\Gamma$-orbit of a point of $X$. Ballmann-Buyalo [BB08, Proposition 1.10] gave the following characterization of groups containing rank one elements in terms of the Tits radius of the limits set:

Proposition 9.5. $\Gamma$ contains no rank one element if and only if $\Lambda(\Gamma)$ lies in the Tits ball of radius $\leq \pi$ centered at a point of $\Lambda(\Gamma)$.

Combining this characterization with results of Schroeder BGS85, Appendix $3]$ one can answer Question 9.4 when every component of the Tits boundary of $X$ has radius $\leq \frac{\pi}{2}$ :

Corollary 9.6. If every component of $X(\infty)$ equipped with the Tits metric has radius $\leq \frac{\pi}{2}$, then a subgroup $\Gamma \leq \operatorname{Iso}(X)$ either contains a rank one element or fixes a point at infinity.

Proof. Following [BGS85, page 220], for a subset $Q \subset X(\infty)$, let

$$
C_{Q}=\left\{z \in Q \mid Q \text { lies in the Tits ball of radius } \frac{\pi}{2} \text { centered at } z\right\} \text {. }
$$

Lower semicontinuity of the Tits distance implies that if $Q$ is closed in the cone topology on $X(\infty)$, then so is $C_{Q}$ [BGS85, 4.9]. If $C_{Q}$ is nonempty, then clearly it has Tits diameter $\leq \frac{\pi}{2}$.

Apply this to $Q=\Lambda(\Gamma)$, which is a closed $\Gamma$-invariant subset. Since $\Gamma$ has no rank one element, Proposition 9.5 implies that $\Lambda(\Gamma)$ lies in the Tits ball of radius $\leq \pi$ about one of its points, and by our assumption the ball must have radius $\leq \frac{\pi}{2}$ (for Tits metric is length so the distance between different components is infinite). Thus $C_{\Lambda(\Gamma)}$ is a closed subset of Tits diameter $\leq \frac{\pi}{2}$.

By the main result of [BGS85, Appendix 3] any subset of $X(\infty)$ that is closed in the cone topology and has Tits diameter $\leq \frac{\pi}{2}$ has a unique center, defined as the center of the closed (Tits) ball of the smallest radius among all balls containing the subset. Let $z_{G}$ be the unique center of $C_{\Lambda(\Gamma)}$. Since $\Lambda(G)$ is $\Gamma$-invariant, so is $C_{\Lambda(\Gamma)}$, and hence $\Gamma$ fixes $z_{G}$. 
Example 9.7. The components of the Tits boundary are points if (and only if) $X$ is visibility [BGS85, 4.14], so Corollary [9.6 applies if $X$ is visibility, in which case one can say more:

Corollary 9.8. If $X$ is visibility and $\Gamma$ contains a parabolic element but no rank one elements, then $\Gamma$ contains no axial isometries, $\Lambda(\Gamma)$ is a point, the fixed point set of $\Gamma$ at infinity equals $\Lambda(\Gamma)$, and $\Gamma$ stabilizes every horoball centered at $\Lambda(\Gamma)$.

Proof. Since $\Gamma$ contains no rank one elements, Proposition 9.5 implies that $\Lambda(\Gamma)$ is a point (as the Tits distance between any two distinct points is infinite). Being a visibility space, $X$ has no flat half spaces, so $\Gamma$ contains no axial isometries. The limit set is $\Gamma$-invariant, so $\Lambda(\Gamma)$ is a fixed point of $\Gamma$. If $\Gamma$ fixed any other point, it would also be fixed by the cyclic subgroup generated by a parabolic in $\Gamma$, but the fixed point set of any abelian subgroup containing a parabolic has Tits radius $\leq \frac{\pi}{2}$, which again is a single point. Thus $\Lambda(\Gamma)$ is a unique fixed point of $\Gamma$.

Remark 9.9. By Corollary 9.8 and Proposition 8.3 any non-virtually-cyclic, discrete isometry group of a visibility manifold that fixes a point at infinity must stabilize a horoball. There is a sizable class of groups to which this applies, e.g. by Corollary 11.6 it contains the product of any nontrivial torsion-free groups, see [KN04] for more examples.

\section{Groups whose Center contains a parabolic}

The following result is implicit in BGS85, Lemma 7.3, 7.8].

Lemma 10.1. $\Gamma \leq I s o(X)$ stabilizes a horoball if it has a finite index subgroup $\Gamma_{0}$ whose center $Z\left(\Gamma_{0}\right)$ contains a parabolic isometry.

Proof. Fix a parabolic isometry $z \in Z\left(\Gamma_{0}\right)$, and right coset representatives $g_{1}, \ldots g_{k}$ of $\Gamma_{0}$ in $\Gamma$. Then the function $x \rightarrow \sum_{i} d\left(g_{i}^{-1} z g_{i} x, x\right)$ is convex and $\Gamma$-invariant. Since $x \rightarrow d(z x, x)$ does not assume its infimum, neither does the above convex function. Then a limiting process outlined in BGS85, Lemma 3.9], cf. [BH99, Lemma II.8.26], gives rise via Arzela-Ascoli theorem to a $\Gamma$ invariant Busemann function, whose sublevel sets are $G$-invariant horoballs.

Flat torus theorem [BH99, Chapter II.7] restricts a discrete isometry group of $X$ whose center consists of axial elements, which can be summarized as follows, see [Bel].

Theorem 10.2. Let $G$ be a group with subgroups $H, G_{0}$ such that their centers $Z(H), Z\left(G_{0}\right)$ are infinite, $Z(H) \subseteq Z\left(G_{0}\right)$, the index of $G_{0}$ in $G$ is finite, and one of the following conditions hold: 
(1) $Z(H)$ is not finitely generated;

(2) any homomorphism $H \rightarrow \mathbb{R}$ is trivial.

(3) $H$ is finitely generated, and $Z(H)$ contains a free abelian subgroup that is not a direct factor of any finite index subgroup of $H$.

If a discrete subgroup of Iso $(X)$ is isomorphic to $G$, then it stabilizes a horoball.

The reader may want to first think through the case when $H=G_{0}=G$, and then go on to observe that if Theorem 10.2 holds for $H, G_{0}, G$, then it also does for $H, G_{0} \times K, G \times K$ for any group $K$.

Example 10.3. Theorem 10.2(1) applies, e.g. to any infinitely generated, torsion-free, countable abelian group of finite rank, such as $(\mathbb{Q},+)$, where finiteness of rank ensures finiteness of cohomological dimension Bie81, Theorem 7.10].

Remark 10.4. It is unknown whether there is a group of type $F$ with infinitely generated center. Such a group cannot be elementary amenable [Bel], or linear over a field of characteristic zero [AS82, Corollary 5]. Sanity check: there does exist a finitely presented group with solvable word problem whose center contains every countable abelian group [OH07, Corollary 3].

Example 10.5. (groups of type $F$ to which Theorem[10.2(3) applies, see Bel]):

(1) $H$ is the fundamental group of the total space of any principal circle bundle with non-zero rational Euler class and a finite aspherical cell complex as the base.

(2) $H$ is a torsion-free, finitely generated, non-abelian nilpotent group.

(3) $H$ is the fundamental group of any closed orientable Seifert 3-manifold modelled on $\widetilde{S L}_{2}(\mathbb{R})$.

(4) $H$ is the preimage of any torsion-free lattice in $S p_{2 n}(\mathbb{R})$ under the universal cover $\widetilde{S p}_{2 n}(\mathbb{R}) \rightarrow S p_{2 n}(\mathbb{R})$ for $n \geq 2$.

(5) $H$ is the amalgamated product $G_{1} *_{A} G_{2}$ where $G_{1}, G_{2}$ have type $F$ and are finitely generated, $A$ lies in the center of $G_{1}, G_{2}$ and contains a subgroup that is not a virtual direct factor of $G_{1}$.

\section{ANCHORED GROUPS AND FIXED POINTS AT INFINITY}

Let us discuss algebraic conditions that force an isometry group of $X$ to fix a point at infinity.

If a subgroup $\Gamma \leq \operatorname{Iso}(X)$ contains a parabolic element, stabilizes a closed convex noncompact subset $W \subseteq X$, and fixes a point at infinity of $W$, then we say that $\Gamma$ is anchored in $W$. (Passing to an invariant closed convex subset is essential in some inductive arguments, e.g. in Theorem [11.7). 
Theorem 11.1. Let $\Gamma \leq I s o(X)$ be a subgroup and $W$ be a any closed, convex, noncompact $\Gamma$-invariant subset of $X$. Then $\Gamma$ is anchored in $W$ if one of the following holds:

(1) $\Gamma$ is abelian and contains a parabolic.

(2) $\Gamma$ has a normal subgroup that is anchored in $W$.

Proof. (1) For $W=X$ this is due to Schroeder [BGS85, Appendix 3], see also cf. [FNS06]. The general case follows from a result of Caprace-Lytchak CL10, Corollary 1.5] that the centralizer of a parabolic isometry of a CAT(0) space of finite telescopic dimension has a fixed point at infinity. Closed convex subsets of Hadamard manifolds have finite telescopic dimension, see [CL10, Section 2.1], and a parabolic isometry of $X$ that stabilizes a closed convex subset acts in that subset as a parabolic isometry.

(2) For $W=X$ this is due to Eberlein [Ebe96, Proposition 4.4.4], whose proof generalizes to our setting via [FNS06, Proposition 5.7] or [BL05, Proposition $1.4]$.

Given a class of Hadamard manifolds $\mathcal{C}$, we say that a group $G$ is clinging in $\mathcal{C}$ if for any discrete subgroup $\Gamma \leq \operatorname{Iso}(Y)$ such that $Y \in \mathcal{C}$ and $\Gamma$ is isomorphic to $G$, and for any $\Gamma$-invariant closed convex noncompact subset $W$ of $Y$, the group $\Gamma$ is anchored in $W$. If $G$ is clinging in the class of all Hadamard manifolds, we simply call $G$ clinging.

In particular, $G$ is clinging in $\mathcal{C}$ if no such $\Gamma$ exists but we of course are interested in nontrivial examples. Theorem 11.2 and Corollary 11.3 below can be found in [Bel].

Theorem 11.2. A group $G$ is clinging in $\mathcal{C}$ if one of the following is true:

(1) $G$ has a clinging in $\mathcal{C}$ normal subgroup, or

(2) $G$ is the union of a nested sequence of clinging in $\mathcal{C}$ subgroups.

(3) $G$ is as in Theorem 10.2.

(4) $G$ is virtually solvable and not virtually- $\mathbb{Z}^{k}$ for any $k$.

(5) a normal abelian subgroup of $G$ contains an infinite $G$-conjugacy class.

Corollary 11.3. Let $G$ be a finitely generated, torsion-free group that has a nontrivial, normal, elementary amenable subgroup. Then either $G$ is clinging, or $G$ has a nontrivial, finitely generated, abelian, normal subgroup that is a virtual direct factor of $G$.

Splitting results of Schroeder Sch85] and Monod Mon06b] give another source of groups fixing points at infinity.

Theorem 11.4. Let $W$ be a closed, convex, noncompact subset of a Hadamard manifold. If a discrete torsion-free isometry group of $W$ contains two commuting subgroups $\Gamma_{1}, \Gamma_{2}$, then one of them fixes a point at infinity of $W$. 
Proof. Suppose neither $\Gamma_{1}$ nor $\Gamma_{2}$ fixes a point at infinity of $W$, and in particular $\Gamma_{1} \Gamma_{2}$ is nontrivial. Since $\Gamma_{1}$ fixes no point at infinity, it follows from Mon06b, Proposition 27, Remark 39 and Subsection 4.6] that $W$ contains a non-empty minimal convex closed $\Gamma_{1}$-invariant subset $C_{1}$, and moreover, the union $C$ of such sets splits as $C_{1} \times C_{2}$ for some bounded convex subset $C_{2}$ where $\Gamma_{1}, \Gamma_{2}$ preserve the splitting and act trivially on $C_{2}, C_{1}$, respectively. (Boundedness of $C_{2}$ is a key point, so we explain it here: if $C_{2}$ is unbounded, it contains a ray $s \rightarrow r(s)$, so given $x \in C_{1}$ we get a ray $\{x\} \times r$ in $X$ which is mapped by any $\gamma \in \Gamma_{1}$ to an asymptotic ray as $\gamma$ maps $(x, r(s))$ to $(\gamma(x), r(s))$; thus $\Gamma_{1}$ fixes a point at infinity contradicting the assumptions). Since $C_{2}$ is bounded, $\Gamma_{2}$ fixes the circumcenter of $C_{2}$, and hence fixes a point $z_{2} \in C$. Repeating the same argument with $\Gamma_{2},\left\{z_{2}\right\}$ in place of $\Gamma_{1}, C_{1}$ shows that the union $Z$ of minimal convex closed $\Gamma_{2}$-invariant subsets splits as the product of $\left\{z_{2}\right\}$ and a bounded convex subset, and the splitting is invariant under $\Gamma_{1}, \Gamma_{2}$. It follows that $\Gamma_{1} \Gamma_{2}$ fixes a point of $Z$. This is where we need that $\Gamma_{1} \Gamma_{2}$ lies in a torsion-free discrete subgroup, because it implies that $\Gamma_{1} \Gamma_{2}$ is trivial.

Corollary 11.5. If $G_{1}$ and $G_{2}$ are groups each containing a subgroup as in Theorem 4.4 (2), then $G_{1} \times G_{2}$ is clinging in the class of all Hadamard manifolds.

Proof. Suppose $G_{1} \times G_{2}$ is isomorphic to a discrete subgroup $\Gamma \leq \operatorname{Iso}(X)$ stabilizing a closed, convex, noncompact subset $W$. By Theorem 4.4(2), each factor contains a parabolic, and one of them fixes a point at infinity by Corollary 11.4, and hence is anchored in $W$. So $\Gamma$ is anchored in $W$ by Theorem 11.1(2).

Corollary 11.6. If $G_{1}, G_{2}$ are nontrivial torsion-free groups, then $G_{1} \times G_{1}$ is clinging in the class of Hadamard manifolds containing no flat half planes.

Proof. Suppose $G_{1} \times G_{2}$ is realized as a discrete isometry group of a Hadamard manifold with no flat half planes, and suppose $W$ is a closed, convex, noncompact invariant subset. Since $G_{1} \times G_{2}$ is torsion-free, by Theorem 11.4 one of the factors fixes a point at infinity of $W$. If say $G_{1}$ contains a hyperbolic element $h$, then $h$ has rank one as $X$ contains no flat half planes, so the centralizer of $h$ in $G_{1} G_{2}$ is cyclic, and also contains $h$ and $G_{2}$ violating the assumption that $G_{2}$ is nontrivial. Thus $G_{1}$ consists of parabolics, and by symmetry so does $G_{2}$. One of the groups $G_{1}, G_{2}$ fixes a point at infinity, hence it is anchored in $W$, and so is $G_{1} G_{2}$ by Theorem 11.1(2).

Results of Caprace-Monod imply:

Theorem 11.7. If $H$ is clinging in a class of Hadamard manifolds $\mathcal{C}$, and $G$ contains $H$ as a co-amenable subgroup, then $G$ is clinging in $\mathcal{C}$. 
Proof. Realize $G$ as a discrete isometry group of a Hadamard manifold in $\mathcal{C}$ stabilizing a closed, convex, noncompact subset $W$. By assumption $H$ contains a parabolic, hence so does $G$. If $G$ does not fix a point at infinity of $W$, then by CM09b, Theorem 4.3] $W$ contains a minimal closed convex $G$-invariant subspace $U$. Note that $U$ has no Euclidean de Rham factor (as the other factor would then be a smaller $\Gamma$-invariant subset). The co-amenability implies that $H$ fixes no point at infinity of $U$ CM09a, Proposition 2.1] so it is not clinging in $\mathcal{C}$.

Remark 11.8. Burger-Schroeder [BS87b] showed that any amenable subgroup $\Gamma \leq \operatorname{Iso}(X)$ either fixes a point at infinity or stabilizes a flat, and this generalizes to actions on proper CAT(0) spaces by Adams-Ballmann [AB98. Even more generally, Caprace-Monod [CM09a, Corollary 2.2] obtained the same conclusion whenever $\Gamma$ contains two commuting co-amenable subgroups (and also gave examples with non-amenable $\Gamma$ ). To make this result into a source of groups that fix a point at infinity more examples are needed, and with our focus on manifolds one has to answer the following.

Question 11.9. Is there a group that contains two commuting co-amenable subgroups, has finite cohomological dimension, and is not virtually solvable?

As mentioned in Section 9, if $\Gamma$ fixes a point at infinity, then $\Gamma$ permutes horospheres centered at the point defining a homomorphism $\Gamma \rightarrow \mathbb{R}$. Thus if $\Gamma \leq I s o(X)$ has no nontrivial homomorphism into $\mathbb{R}$, then $\Gamma$ stabilizes a horoball if and only if $\Gamma$ fixes a point at infinity.

Examples of clinging groups with finite abelianization (and hence no nontrivial homomorphisms into $\mathbb{R}$ ) are abound, see [Bel], and there are many such groups of finite cohomological dimension, or even of type $F$ (so they may well be the fundamental groups of complete manifolds of $K \leq 0$ ).

Note that the property of having finite abelianization is inherited by amalgamated products (clearly), and by extensions (due to right exactness of the abelianization functor). Moreover, an extension with a finite quotient often has finite abelianization, e.g. the abelianization of the semidirect product $A \rtimes B$ is $\left(A^{\mathrm{ab}}\right)_{B} \times B^{\mathrm{ab}}$, where $\left(A^{\mathrm{ab}}\right)_{B}$ is the coinvariants for the $B$-action on $A^{\mathrm{ab}}$.

\section{Homotopy obstructions (after Gromov and Izeki-Nayatani)}

If $M$ is a complete manifold of $K \leq 0$, then $\pi_{1}(M)$ has finite cohomological dimension. A group has finite cohomological dimension if and only if it is the fundamental group of a manifold whose universal cover is diffeomorphic to a Euclidean space.

Gromov asked Gro93] whether every countable group of finite cohomological dimension is isomorphic to some $\pi_{1}(M)$ where $M$ is complete of $K \leq 0$. (The 
question is a good illustration of how little we know about open manifolds of $K \leq 0$.) The answer is no due to groundbreaking works of Gromov Gro03. and Izeki-Nayatani [IN05] on groups with strong fixed point properties.

These papers combine certain averaging procedures with ideas of harmonic map superrigidity to produce many a group $G$ such that

(a) any isometric $G$-action on a Hadamard manifold has a fixed point;

(b) $G$ has type $F$ (i.e. is the fundamental group of a compact aspherical manifold with boundary).

Since the $\pi_{1}(M)$-action on the universal cover of $M$ is free, it follows that there is no complete manifold $M$ of $K \leq 0$ and $\pi_{1}(M) \cong G$. The methods actually reach far beyond Hadamard manifolds, and apply to isometric $G$-actions on a wide variety of spaces, see [IN10, NS11, IKN12].

Gromov's examples are certain torsion-free hyperbolic groups produced from a sequence of graphs $\Gamma_{n}$ whose edges are labeled with words of length $j$ in an alphabet of $d>1$ letters. The words are chosen randomly, and reversing orientation of the edge corresponds to taking inverse of a word. Given the data let $G\left(\Gamma_{n}, d, j\right)$ be the quotient group of $F_{d}$, the free group on $d$ generators, by the relations corresponding to the cycles in $\Gamma_{n}$. The main result is that there is a sequence of expander graphs $\Gamma_{n}$ such that for a large enough $j$ the group $G\left(\Gamma_{n}, d, j\right)$ is torsion-free, hyperbolic, and satisfies (a) with probability $\rightarrow 1$ as $n \rightarrow \infty$. Like any torsion-free hyperbolic group, $G\left(\Gamma_{n}, d, j\right)$ has type $F$.

Izeki-Nayatani's original example is any uniform torsion-free lattice in $P S L_{3}\left(\mathbb{Q}_{p}\right)$, which has type $F$ because it acts freely and properly discontinuously on the associated Euclidean building.

Remark 12.1. The class of groups that satisfy (a) includes any finite group, or more generally any locally finite infinite subgroup such as $\mathbb{Q} / \mathbb{Z}$. There are much deeper examples in $\left[\mathrm{ABJ}^{+} 09\right]$ of finitely presented, infinite, non-torsion-free groups that fix a point for any action by a homeomorphisms on a contractible manifold. None of these groups satisfies (b) as they have nontrivial finite order elements.

Remark 12.2. By Davis's reflection group trick any group of type $F$ embeds into the fundamental group of a closed aspherical manifold. Thus there is a closed aspherical manifold that is not homotopy equivalent to a complete manifold of $K \leq 0$.

Question 12.3. Is there a closed aspherical manifold whose fundamental group satisfies (a)? 


\section{HomeOmorphism OBSTRUCTIONS: EXPLOITING $\mathbb{R}$ FACTORS}

Call a group $G$ reductive if for any epimorphism $G \rightarrow H$ such that $H$ is a discrete, non-cocompact, torsion-free isometry group of a Hadamard manifold stabilizing a horoball, or a totally geodesic submanifold where $H$ acts cocompactly.

Example 13.1. (1) Any quotient of a reductive group is reductive.

(2) Any finitely generated, virtually nilpotent group is reductive (as follows from Sections 4, 10, see [Bel]).

(3) Any irreducible, uniform lattice in the isometry group of a symmetric space of $K \leq 0$ and real rank $>1$ is reductive, by the harmonic map superrigidity [Duc, Theorem 1.2], see also [Bel].

A manifold is covered by $\mathbb{R}^{n}$ if its universal cover is diffeomorphic to $\mathbb{R}^{n}$. Thus any complete $n$-manifold of $K \leq 0$ is covered by $\mathbb{R}^{n}$.

Remark 13.2. It is well-known that an open $K(G, 1)$ manifold covered by $\mathbb{R}^{n}$ exists if and only if $G$ is a countable group of finite cohomological dimension, see e.g. [Bel].

A manifold is covered by $\mathbb{R} \times \mathbb{R}^{n-1}$ if it is diffeomorphic to the product of $\mathbb{R}$ and a manifold covered by $\mathbb{R}^{n-1}$. For instance, if $G$ is a discrete torsionfree isometry group of a Hadamard manifold that satisfies the condition (3) of Section 3, then $M$ is covered by $\mathbb{R} \times \mathbb{R}^{n-1}$. As we saw above (3) can be forced by purely algebraic assumptions on $\pi_{1}(M)$ :

Example 13.3. If $M$ is a complete connected manifold of $K \leq 0$ such that $\pi_{1}(M)$ is either clinging with finite abelianization, or satisfies the assumptions of Theorem 10.2 , then $M$ is covered by $\mathbb{R} \times \mathbb{R}^{n-1}$.

A trivial method of producing manifolds that are covered by $\mathbb{R}^{n}$ but not covered by $\mathbb{R} \times \mathbb{R}^{n-1}$ is to consider any manifold of minimal dimension among all manifolds in its homotopy type that are covered by a Euclidean space, which yields (see $[\mathrm{Bel}]$ ):

Proposition 13.4. Any aspherical manifold is homotopy equivalent to a manifold covered by $\mathbb{R}^{n}$ but not covered by $\mathbb{R} \times \mathbb{R}^{n-1}$.

This method is non-constructive for it is not easy to decide whether a specific open manifold has the minimal dimension in the above sense (see BKK02, BF02a, Yoo04, Des06] for the manifolds of such minimal dimensions).

Corollary 13.5. If $G$ is reductive, clinging with finite abelianization, or as in Theorem 10.2, then any $K(G, 1)$ manifold is homotopy equivalent to a manifold that admits no metric of $K \leq 0$ and is covered by a Euclidean space. 
Example 13.6. Corollary 13.5 applies if $G=\mathbb{Q}$, see Example 10.3 ,

An essential tool in understanding manifolds covered by $\mathbb{R} \times \mathbb{R}^{n-1}$ is the recent result of Guilbault Gui07]: if an open manifold $W$ of dimension $\geq 5$ is homotopy equivalent to a finite complex, then $\mathbb{R} \times W$ is diffeomorphic to the interior of a compact manifold. Building on this result, the author [Bel] proved

Theorem 13.7. Let $W$ be an open $(n-1)$-manifold with $n \geq 5$ that is homotopy equivalent to a finite complex of dimension $k \leq n-3$. Then $\mathbb{R} \times W$ is diffeomorphic to the interior of a regular neighborhood of a $k$-dimensional finite subcomplex.

With more work one gets [Bel] the following applications:

Theorem 13.8. Let $L$ be a finite aspherical $C W$ complex such that $G=\pi_{1}(L)$ is reductive, clinging with finite abelianization, or as in Theorem 10.2. Suppose that $L$ is homotopy equivalent to a complete $n$-manifold $M$ of $K \leq 0$ and $n \geq 5$, and set $l=\operatorname{dim}(L)$.

(1) If $l \leq n-3$, then $M$ diffeomorphic to the interior of a regular neighborhood of a k-dimensional finite subcomplex.

(2) If $L$ is a closed manifold of dimension $<\frac{2 n-2}{3}$, then $M$ is diffeomorphic to the total space of a vector bundle over $L$.

(3) If $l<\frac{n}{2}$, then every complete $n$-manifold of $K \leq 0$ in the tangential homotopy type of $M$ is diffeomorphic to $M$.

(4) If $l \leq n-3$, then the tangential homotopy type of $M$ contains countably many open $n$-manifolds that admit no complete metric of $K \leq 0$.

Question 13.9. Can one strengthen the conclusion "countably many" in the part (4) of Theorem 13.8 to "a continuum of"?

A positive answer is given in Bel] under a technical assumption which holds e.g. if $L$ is a closed manifold, or if either $\mathbb{Z}^{3}$ or $\mathbb{Z} * \mathbb{Z}$ does not embed into $G$.

Remark 13.10. Limiting Theorem 13.8 to certain classes of manifolds of $K \leq 0$ may result in enlarging the class of allowable fundamental groups, e.g. applying the theorem to manifolds with visibility universal cover, we can allow $G$ to be the product of any two nontrivial groups.

As an application of Theorem 13.7, we get the following characterization of $\mathbb{R}^{n}$ :

Corollary 13.11. An open contractible $n$-manifold $W$ is homeomorphic to $\mathbb{R}^{n}$ if and only if $W \times S^{1}$ admits a metric of $K \leq 0$.

If $n=4$, then "homeomorphic" in Corollary 13.11 cannot be upgraded to "diffeomorphic": if $W$ is an exotic $\mathbb{R}^{4}$, then $W \times S^{1}$ is diffeomorphic to $\mathbb{R}^{4} \times S^{1}$. 


\section{Benefits of A LOWER CURVAture Bound}

Complete manifolds of Ric $\geq-(n-1)$ are central to the global Riemannian geometry. For manifolds of $K \leq 0$, a lower Ricci curvature bound at a point is equivalent (by standard tensor algebra considerations) to a lower sectional curvature bound at the same point; by rescaling one can always make the bounds equal the curvature of the hyperbolic $n$-space.

In the seminal work Gro82] Gromov uncovered a relation between the simplicial volume and volume growth, which for complete manifolds with Ric $\geq-(n-1)$ is governed by Bishop-Gromov volume comparison. The following can be found in [Gro82, p.13, 37].

Theorem 14.1. (Gromov) Let $W$ be an $n$-manifold such that every component $C_{i}$ of $\partial W$ is compact. If the interior of $W$ is homeomorphic to a complete manifold of Ric $\geq-(n-1)$, then $\sum_{i}\left\|C_{i}\right\| \leq \liminf _{r \rightarrow \infty} \frac{\operatorname{Vol} B_{p}(r)}{r}$.

Here $B_{p}(r)$ is the $r$-ball in $M$ centered at $p$, and $\left\|C_{i}\right\|$ is the simplicial volume of $C_{i}$.

Example 14.2. If $M$ in Theorem 14.1 has finite volume, or more generally sublinear volume growth, then each component of $\partial W$ has zero simplicial volume.

Question 14.3. Does every complete manifold with $-1 \leq K \leq 0$ and sublinear volume growth admit a finite volume metric?

Example 14.4. Any infinite cyclic cover a closed connected manifold $L$ of $K \leq 0$, i.e. the cover corresponding to the kernel of a surjective homomorphism $\pi_{1}(L) \rightarrow \mathbb{Z}$, has linear growth.

Problem 14.5. Study manifolds of $-1 \leq K \leq 0$ with linear volume growth.

Another consequence of a lower curvature bound is the famous Margulis lemma which appeared in BGS85] for manifolds of $-1 \leq K \leq 0$ following unpublished ideas of Margulis, and in [FY92, KPT10] for manifolds of $K \geq-1$. The following version of the Margulis lemma for manifolds of Ric $\geq-(n-1)$ is due to Kapovitch-Wilking [KW] with essential ingredients provided by prior works of Cheeger-Colding.

Theorem 14.6. (Kapovitch-Wilking) For each $n$ there are constants $m$ and $\varepsilon \in(0,1)$ such that if $p$ is a point of a complete $n$-manifold with Ric $\geq-(n-1)$ on $B_{p}(1)$, then the image of $\pi_{1}\left(B_{p}(\varepsilon)\right) \rightarrow \pi_{1}\left(B_{p}(1)\right)$ has a nilpotent subgroup generated by $n$ elements and of index $\leq m$. 
In fact, the nilpotent subgroup in Theorem 14.6 has a generated set $\left\{s_{1}, \ldots, s_{n}\right\}$ such that $s_{1}$ is central and the commutator $\left[s_{i}, s_{j}\right]$ is contained in the subgroup generated by $s_{1}, \ldots, s_{i-1}$ for each $1<i<j$. Another universal bound on the number of generators of any given $r$-ball is given by

Theorem 14.7. (Kapovitch-Wilking) For each $n, r$ there is a constant $k$ such that if $p$ is a point in a complete Riemannian manifold $M$ such that $\pi_{1}\left(B_{p}(r)\right) \rightarrow \pi_{1}(M, p)$ is onto and Ric $\geq-(n-1)$ on $B_{p}(4 r)$, then $\pi_{1}(M, p)$ is generated by $\leq k$ elements.

An important feature of the two preceding results is that no curvature control is required outside a compact subset.

\section{INJECTIVITY RADIUS GOING TO ZERO AT INFINITY}

We say that a subset $S$ of a Riemannian manifold has $\operatorname{Inj} R a d \rightarrow 0$ if and only if for every $\varepsilon>0$ the set of points of $S$ with injectivity radius $\geq \varepsilon$ is compact; otherwise, we say $S$ has $\operatorname{Inj} \operatorname{Rad} \not \rightarrow 0$.

Remark 15.1. By volume comparison any finite volume complete manifold of $K \leq 0$ has Inj Rad $\rightarrow 0$ [BGS85, 8.4].

Proposition 15.2. Any finite volume complete real hyperbolic manifold admits a complete metric with Inj Rad $\rightarrow 0$, bounded negative curvature, infinite volume, and sublinear volume growth.

Proof. We just do the two dimensional case; the general case is similar. Any end of a finite volume complete hyperbolic surface surface has an annular neighborhood with the metric $d t^{2}+e^{-2 t} d \phi^{2}, t>0$. Modify it to the metric $d t^{2}+f^{2}(t) d \phi^{2}$ where $f$ is a convex decreasing function such that $f(t)=e^{-t}$ for small $t$, and $f(t)=t^{-\alpha}, \alpha \in(0,1)$ for large $t$. Let $\Sigma_{f}$ be the resulting complete Riemannian 2-manifold, and let $\Sigma_{f}^{r}$ denote " $\Sigma_{f}$ with the portion with $t>r$ chopped off". Now $\Sigma_{f}$ has

- Inj Rad $\rightarrow 0$ because $f$ monotonically decreases to zero,

- infinite volume since $\frac{1}{2 \pi} \operatorname{Vol}\left(\Sigma_{f}^{r}\right)$ grows (sublinearly) as $\int_{0}^{r} f(s) d s=\frac{r^{1-\alpha}}{1-\alpha}$,

- bounded negative sectional curvature because on the annular neighborhood $K=-\frac{f^{\prime \prime}}{f}<0$ which equals $-\frac{\alpha(\alpha+1)}{t^{2}}$ for $t>r$.

Question 15.3. Is there a complete manifold of $K \leq 0$ and Inj Rad $\rightarrow 0$ that admits no complete finite volume of $K \leq 0$ ? What is the answer in the presence of a lower curvature bound.

Gromov Gro78] pioneered the study of ends of negatively curved manifolds via the critical point theory for distance functions, which was extended by Schroeder [BGS85, Appendix 2] as follows: 
Theorem 15.4. (Schroeder) If $M$ is a complete manifold of Inj Rad $\rightarrow 0$ and $-1 \leq K \leq 0$, then either $M$ is the interior of a compact manifold, or $M$ contains a sequence of totally geodesic, immersed, flat tori with diameters approaching zero.

None of the assumptions in the above theorem can be dropped due to examples of Gromov [BGS85, Chapter 11] and Nguyen Phan [NPb] in which $\pi_{1}(M)$ is infinitely generated, see Section 20.

Problem 15.5. Find a geometrically meaningful compactification of complete manifolds of Inj $\operatorname{Rad} \rightarrow 0$ and $-1 \leq K \leq 0$.

In the locally symmetric case this was accomplished in Leu95, Sap97, Leu04, and pinched negatively curved manifolds are naturally compactified by horospheres.

A weak substitute for a geometrically meaningful compactification is given by the following general theorem CG91:

Theorem 15.6. (Cheeger-Gromov) For each $n$ there is a constant $c$ such that any complete finite volume $n$-manifold $M$ of $|K| \leq 1$ admits an exhaustion by compact smooth codimension zero submanifolds $M_{i}$ with boundary such that $M_{i} \subset \operatorname{Int}\left(M_{i+1}\right)$, the norm of the second fundamental form of $\partial M_{i}$ is $\leq c$, and $\operatorname{Vol}\left(\partial M_{i}\right) \rightarrow 0$ as $i \rightarrow \infty$.

The proof constructs a controlled exhaustion function on $M$. For a related work based on different technical tools see [SY94, Theorem I.4.2] and [Daf97, WL97].

If $M$ in Theorem 15.6 is the interior of a manifold $W$ with compact boundary, then considering the components of $\partial M_{i}$ that lie in a collar neighborhood of $\partial W$, we conclude that

Corollary 15.7. If $W$ is a manifold with compact connected boundary whose interior admits a complete finite volume metric $g$ of $|K| \leq 1$, then $\mathbb{R} \times \partial W$ contains the sequence of compact separating hypersurfaces $H_{i}$ which are homologous to $\{0\} \times \partial W$ and satisfy $\operatorname{Min} \operatorname{Vol}\left(H_{i}\right) \rightarrow 0$ as $i \rightarrow \infty$. Moreover,

(1) $\partial W$ has even Euler characteristic and zero simplicial volume;

(2) If $\partial W$ is orientable, then its Pontryagin numbers vanish.

(3) If $g$ also has $K \leq 0$, then the $\ell^{2}$-Betti numbers of $\partial W$ vanish, and hence $\partial W$ has zero Euler characteristic.

Proof. Projecting onto the $\partial W$ factor yields a degree one map $\partial M_{i} \rightarrow \partial W$ (if $\partial W$ is non-orientable, then so is $H_{i}$, and we get a degree one map of their orientation covers). Thus $\partial W$ has zero simplicial volume, which of course we already knew by Theorem 14.1 . 
By Chern-Weil theory the Pontryagin numbers $p_{I}(L)$ of a closed manifold $L$ satisfy $\left|p_{I}(L)\right| \leq c_{l} \operatorname{Min} \operatorname{Vol}(L)$ where $c_{l}$ is a constant depending only on $l=$ $\operatorname{dim}(L)$ Gro82. Thus $p_{I}\left(H_{i}\right) \rightarrow 0$ as $i \rightarrow \infty$. Since Pontryagin numbers are oriented cobordism invariant, we conclude that if $\partial W$ in Corollary 15.7 is orientable, then its Pontryagin numbers vanish.

The boundary of a compact manifold has even Euler characteristic [Dol95, Corollary VIII.8.8], so applying this to the cobordism between $\partial W$ and $H_{i}$ we see that $\chi(\partial W)+\chi\left(H_{i}\right)$ is even, and again Chern-Weil theory implies $\chi\left(H_{i}\right) \rightarrow 0$ as $i \rightarrow \infty$, and the claim follows.

Finally, vanishing of the $\ell^{2}$-Betti numbers follows from [CG85, Theorem 1.2], and their alternating sum equals the Euler characteristic.

Question 15.8. Do any of the conclusions Corollary 15.7 hold for complete manifolds with $-1 \leq K \leq 0$ and $\operatorname{Inj} \operatorname{Rad} \rightarrow 0$.

Example 15.9. If $\Sigma_{f}$ is as in Proposition 15.2, then the Riemannian product $\Sigma_{f} \times \Sigma_{f}$ has Inj Rad $\rightarrow 0$ but superlinear volume growth if $0<\alpha \leq \frac{1}{2}$ because for large $r$ the subset $\Sigma_{f}^{r} \times \Sigma_{f}^{r}$ is sandwiched between concentric balls of radii $r$ and $3 r$, and its volume grows superlinearly as $\alpha \leq \frac{1}{2}$. Thus proving that the boundary has zero simplicial volume one requires new ideas beyond Theorem 14.1.

\section{NeGATIVELY CURVED MANIFOLDS With UNIFORM VOLUME BOUND}

For a connected complete Riemannian manifold $M$ we denote by $\widetilde{M}$ its universal cover with the pullback metric.

Fukaya Fuk84 proved the following result, whose analog for closed manifolds of dimension $\neq 3$ is due to Gromov [Gro78]:

Theorem 16.1. (Fukaya) Given $V$ and $n \neq 3,4$, only finitely many of diffeomorphism classes contain open complete $n$-manifolds $M$ such that

(1) $K<0$ or $\widetilde{M}$ is visibility,

(2) $K \geq-1$

(3) $\operatorname{Vol}(M)<V$.

In dimension four Fukaya proved that the class of manifolds satisfying (1)-(3) contains only finitely many homotopy types (the missing ingredient is the weak h-cobordism theorem, which is unknown for h-cobordisms between closed 3manifolds).

The theorem fails in dimension three as there are infinitely many (both open and closed) hyperbolic 3 -manifolds with uniformly bounded volume Thu. Taking products with flat tori demonstrates that (1) cannot be replaced with $K \leq 0$, even though the optimal curvature condition is unclear. 
Question 16.2. Is Theorem 16.1 true with (1) replaced by " $\widetilde{M}$ has rank one", or " $\widetilde{M}$ contains no flat half planes"?

Remark 16.3. The proof in Gro78, Fuk84 established an upper diameter bound in terms of volume, and then applies Cheeger's finiteness theorem (if $M$ is open the diameter bound is for a compact domain $D$ such that $M \backslash D$ is the interior of an h-cobordism). The strategy fails if one merely assumes that $\widetilde{M}$ has rank one by the following example: Chop off a cusp of a finite volume complete real hyperbolic manifold, and modify the metric to have totally geodesic flat boundary and $K \leq 0$. Then double along the boundary, which gives a finite volume complete rank one manifold of $K \leq 0$ and volume bounded roughly by $2 \mathrm{Vol}(M)$, but its diameter can be chosen arbitrary large by chopping deeper into the cusp.

Question 16.4. How does the number of diffeomorphism types of manifolds $M$ in Theorem 16.1 grows with $n$ and $V$ ?

In the locally symmetric case the above question was extensively studied, see Gel04] and references therein.

\section{NON-ASPHERICAL ENDS OF NONPOSITIVELY CURVED MANIFOLDS}

If a (not necessarily connected) manifold $B$ is diffeomorphic to the boundary of a connected, smooth (not necessarily compact) manifold $W$, then we say that $B$ bounds $W$.

Any aspherical manifold $B$ bounds a noncompact aspherical manifold, namely $B \times[0,1)$, and in fact, the universal cover of $B \times(0,1)$ is a Euclidean space. Note that $B \times(0,1)$ admits a complete metric of $K \leq 0$ if $B$ is an infranilmanifold [BK05], or if $B$ itself admits a complete metric of $K \leq 0$. On the other hand, if $\pi_{1}(B)$ contains a subgroup with strong fixed point properties as in Section 12, then $B \times(0,1)$ admits no complete metric of $K \leq 0$. Our ignorance is illustrated by the following

Question 17.1. Does every closed aspherical manifold bounds a manifold whose interior admits a finite volume complete metric of $K \leq 0$ ?

In this section we discuss similar matters when $B$ is closed and not aspherical. We focus on easy-to-state results and refer to $[\mathrm{BNP}$ for a complete account.

Boundaries of compact manifolds with a complete metric of $K \leq 0$ on the interior could be quite diverse:

\section{Example 17.2.}

(1) The total space of any vector bundle a closed manifold of $K \leq 0$ admits a complete metric of $K \leq 0$ [And87]. 
(2) Complete finite volume locally symmetric manifold of $K \leq 0$ and $\mathbb{Q}$-rank $\geq 3$ are interiors of compact manifolds with non-aspherical boundary.

(3) A complete manifold $M$ of $K \leq 0$ is convex-cocompact if it deformation retracts onto a compact locally convex subset; such $M$ is the interior of a compact manifold whose boundary is often non-aspherical.

There seem to be no simple description of closed manifolds that bound aspherical ones, and some obstructions are summarized below. In order for $B$ to bound an aspherical manifold, a certain covering space of $B$ must bound a contractible manifold. In formalizing how this restricts the topology of $B$, the following definition is helpful: given a class of groups $\mathcal{Q}$, a group is anti- $\mathcal{Q}$ if it admits no nontrivial homomorphism into a group in $\mathcal{Q}$. Clearly, the class of anti- $\mathcal{Q}$ groups is closed under extensions, quotients, and any group generated by a family of anti- $\mathcal{Q}$ subgroups is anti- $\mathcal{Q}$.

Example 17.3. Let $\mathcal{A}_{n}$ denote the class of fundamental groups of aspherical $n$-manifolds. See $\mathrm{BNP}$ for examples of anti- $\mathcal{A}_{n}$ groups in such as:

(1) Any group generated by a set of finite order elements.

(2) Any irreducible lattice in the isometry group of a symmetric space of rank $\geq 2$ and dimension $>n$ BF02a].

The following summarizes some obstructions that prevent a manifold from bounding an aspherical one.

Theorem 17.4. If $B$ bounds an aspherical, non-contractible $n$-manifold, and $\pi_{1}(B)$ is anti- $\mathcal{A}_{n}$, then $B$ is noncompact, parallelizable, its $\mathbb{Z}$-valued intersection form of vanishes, and its $\mathbb{Q} / \mathbb{Z}$-valued torsion linking form vanishes.

Example 17.5. The following manifolds do not bound aspherical ones:

(1) The connected sum of lens spaces, because it is a closed manifold whose fundamental group is anti- $\mathcal{A}_{n}$.

(2) The product of any manifold with $C P^{k}$ with $k \geq 2$.

(3) The connected sum of any manifold and the product of two closed manifolds whose fundamental groups are anti- $\mathcal{A}_{n}$.

(4) The product of a punctured 3-dimensional lens space and a closed manifold whose fundamental group is anti- $\mathcal{A}_{n}$.

(5) Any manifold that contains the manifold in (2)-(4) as an open subset.

Let $\mathcal{N} \mathcal{P}_{n}$ denote the class of the fundamental groups of complete $n$-manifolds of $K \leq 0$; of course $\mathcal{N} \mathcal{P}_{n} \subseteq \mathcal{A}_{n}$. Examples of anti- $\mathcal{N} \mathcal{P}_{n}$ groups of type $F$ discussed in Section 12 immediately imply (see [BNP]):

Theorem 17.6. There is a closed non-aspherical manifold that 
(i) bounds a manifold whose interior is covered by a Euclidean space;

(ii) bounds no manifold whose interior has a complete metric of $K \leq 0$.

Other obstructions come from results of Section 13. Given groups $I, J$ and a class of groups $\mathcal{Q}$ we say that $I$ reduces to $J$ relative to $\mathcal{Q}$ if every homomorphism $I \rightarrow Q$ with $Q \in \mathcal{Q}$ factors as a composite of an epimorphism $I \rightarrow J$ and a homomorphism $J \rightarrow Q$. Here we are mainly interested in groups that reduce relative to $\mathcal{N} \mathcal{P}_{n}$ to the groups from the parts (2)-(3) of Example 13.1, which have finite virtual cohomological dimension.

Theorem 17.7. Let $n \geq 6$, let $G$ be a group from Example 13.1(2)-(3) of virtual cohomological dimension $\leq n-3$, and let $B$ be a closed $(n-1)$-manifold such that $\pi_{1}(B)$ reduces to $G$ relative to $\mathcal{N} \mathcal{P}_{n}$. If $B$ bounds a manifold $N$ such that $\operatorname{Int}(N)$ admits a complete metric of $K \leq 0$, then there is a closed manifold $L$ of dimension $\leq n-3$ such that

(1) $L$ is either an infranilmanifold, or an irreducible, locally symmetric manifold of $K \leq 0$ and real rank $\geq 2$.

(2) $N$ is the regular neighborhood of a PL-embedded copy of $L$.

(3) If $N$ is not diffeomorphic to the product of a compact manifold and a closed interval, then $L$ admits a metric of $K \leq 0$ and $N$ is the total space of a linear disk bundle over $L$.

In BNP we give examples of manifolds $B$ that cannot bound a manifold $N$ as in the above theorem such as

Example 17.8. Let $B$ be the total space of a linear $S^{k}$ bundle over a closed non-flat infranilmanifold such that $k \geq 3$ and the rational Euler class of the bundle is nonzero. Then $B$ does not bound a manifold whose interior admits a complete metric of $K \leq 0$.

A boundary component of a manifold is incompressible if its inclusion induces injections on all homotopy groups. Reductive groups were defined in Section 13 .

Theorem 17.9. Let $B$ be a closed $(n-1)$-manifold such that $\pi_{1}(B)$ is reductive and any nontrivial quotient of $\pi_{1}(B)$ in the class $\mathcal{N} \mathcal{P}_{n}$ has cohomological dimension $n-1$. If $B$ bounds a manifold $N$ such that $\operatorname{Int}(N)$ admits a complete metric of $K \leq 0$, then $B$ is incompressible in $N$.

Example 17.10. Theorem 17.9 applies to whenever $\pi_{1}(B)$ is isomorphic to $\pi_{1}(L)$ where $L$ is an $(n-1)$-manifold of from Theorem 17.7(1) and $\pi_{1}(L)$ has no proper torsion-free quotients. Examples of such $L$ include any higher rank, irreducible, locally symmetric manifolds of $K \leq 0$ (thanks to the Margulis Normal Subgroup Theorem), as well as certain infranilmanifolds, see [BNP]. 
Given a compact boundary component $B$ of a manifold $N$, an end $E$ of $\operatorname{Int}(N)$ that corresponds to $B$ is the intersection of $\operatorname{Int}(N)$ with a closed collar neighborhood of $B$; note that $E$ is diffeomorphic to $[1, \infty) \times B$.

Theorem 17.11. Let $B$ be a closed connected manifold that bounds a manifold $N$, and let $E$ be an end of $\operatorname{Int}(N)$ corresponding to $B$. If $\pi_{1}(B)$ is reductive, and $\operatorname{Int}(N)$ admits a complete metric of $K \leq 0$ and $\operatorname{Inj} \operatorname{Rad} \rightarrow 0$ on $E$, then $B$ is incompressible in $N$.

Example 17.12. If $B$ is the total space of a linear $S^{k}$ bundle with $k \geq 2$ over a manifold $L$ as in 17.7(1), then $B$ does not bound a manifold whose interior admits a complete metric of $K \leq 0$ and Inj Rad $\rightarrow 0$.

\section{Riemannian hyperbolization (after Ontaneda)}

A recent work of Ontaneda Ont allows to dramatically expand the list of known finite volume complete manifolds of $-1 \leq K<0$ of dimensions $>3$. Unlike earlier examples, Ontaneda's method assembles a manifold of $K \leq-1$ in a lego-like fashion from identical blocks according to a combinatorial pattern specified by a cube complex structure on any given manifold. Each block is a compact real hyperbolic manifold with corners, where every boundary face is totally geodesic and the faces's combinatorial pattern is that of a cube. This process results in a singular metric, which Ontaneda is able to smooth into a complete Riemannian metric of $K \leq-1$, provided the block's faces have a sufficiently large normal injectivity radii; such blocks exist.

The idea of building locally CAT(0) manifold out of identical blocks is due to Gromov Gro87] who came up with several hyperbolization procedures turning a simplicial complex into a locally CAT $(0)$ cubical complex. Gromov's ideas were developed and made precise in [DJ91, DJW01, cf. [Dav08, and furthermore Charney-Davis [CD95] developed the strict hyperbolization that turns a cubical complex into a piecewise polyhedral complex whose faces are the blocks of the previous paragraph. A key feature of the procedures is that the link of every vertex of the hyperbolized polyhedral complex is a subdivision of the corresponding link in the original complex, which has two consequences:

- The hyperbolization(s) turns a manifold triangulation into a locally CAT(0) manifold with a cubical complex structure;

- The strict hyperbolization turns a locally CAT(0) cubical complex (or manifold) into a locally CAT $(-1)$ polyhedral complex (manifold, respectively).

Trivial exceptions aside, the resulting piecewise Euclidean (or piecewise hyperbolic) metric is non-Riemannian. Smoothing the piecewise hyperbolic metric into a Riemannian metric of $K \leq-1$ in Ont] is a technological tour de force. 


\section{Remark 18.1.}

(1) The cube complex fed into Ontaneda's construction need not be locally CAT( 0$)$, so the resulting manifolds of $K \leq-1$ in Ont are a priori not homeomorphic to locally CAT $(-1)$ manifolds of [CD95.

(2) Charney-Davis CD95 describe a canonical smoothing of their manifolds (but not of the metrics), yet this smoothing is not necessarily equal to the smooth structure in Ont even when there is a face preserving homeomorphism between the two manifolds.

(3) Taking the normal injectivity radius of the block's faces large enough, one can make the sectional curvature of the resulting manifold arbitrary close to -1 .

(4) If one starts with a closed manifold, then Ontaneda's procedure yields a closed manifold of $K \leq-1$, but if the initial manifold is open, its triangulation contains infinitely many simplices, so the resulting Riemannian manifold of $K \leq-1$ has infinite volume.

In order to produce finite volume examples, Ontaneda relativizes the construction as follows: Start with a closed manifold $B$ that bounds a compact manifold $W$, cone off the boundary, apply the strict hyperbolization, and remove the cone point. The result is a compact manifold with boundary, and Ontaneda gives it a certain smooth structure in which the boundary becomes diffeomorphic to $B$. Topological properties of the resulting smooth manifold $R_{W, B}$ mirror those of $W$, see a summary in [Bel07], and in particular by varying $W$, one finds $R_{W, B}$ 's such that

- $R_{W, B}$ has a nontrivial rational Pontryagin class if $\operatorname{dim}(W) \geq 4$,

- $H^{*}\left(R_{W, B}\right)$ contains a subring isomorphic to the cohomology ring of a given finite CW complex of dimension $<\operatorname{dim}(W) / 2$, see Ont,

- $\pi_{1}\left(R_{W, B}\right)$ surjects on a given finitely presented group if $\operatorname{dim}(W) \geq 4$.

Moreover $R_{W, B}$ enjoys the following properties:

- $R_{W, B}$ is orientable if so is $W$,

- the inclusion of each component of $B$ into $R_{W, B}$ is $\pi_{1}$-injective [DJW01,

- $R_{W, B}$ is aspherical if and only if each component of $B$ is aspherical DJW01,

- $\pi_{1}\left(R_{W, B}\right)$ is hyperbolic relative to the images of the fundamental groups of components of $B$ [Bel07].

The piecewise hyperbolic metric on $R_{W, B}$ is singular and incomplete near the removed cone point, but again when the normal injectivity radius of the block's faces large enough, Ontaneda is able to smooth the metric away from a punctured neighborhood of the cone point, while on that neighborhood the metric 
has to be constructed by an ad hoc method depending on $B$. The following result is implicit in Ont.

Theorem 18.2. (Ontaneda) Let $B$ be the boundary of a compact $n$-manifold and suppose that

(i) if $n \geq 6$, then any $h$-cobordism from $B$ to another manifold is a product;

(ii) $\mathbb{R} \times B$ admits a complete metric $g$ of sectional curvature within $[-1,0)$ such that $(-\infty, 0] \times B$ has finite $g$-volume, and $g=d r^{2}+e^{2 r} g_{B}$ on $[c, \infty) \times B$ for some $c>0$ and a metric $g_{B}$ on $B$.

Then $B$ bounds a compact smooth manifold whose interior admits a complete metric of finite volume and sectional curvature in $[-1,0)$.

Condition (ii) implies that each component of $B$ is aspherical, and hence has torsion-free fundamental group. The Whitehead Torsion Conjecture, which is true for many groups of geometric origin BL12, BFL14, predicts that all torsion-free groups have zero Whitehead torsion. If the conjecture is true for the fundamental group of each component of $B$, then (i) holds. Condition (ii) has been checked in a number of cases in BK05, NPa, Bel13 implying:

Theorem 18.3. A manifold $B$ bounds a compact manifold whose interior admits a complete metric of finite volume and sectional curvature in $[-1,0)$ if

(1) $B$ is a closed 3-dimensional Sol manifolds [NPa, or

(2) $B$ bounds a compact manifold, and belongs to the class $\mathcal{B}$ [Bel13].

Here $\mathcal{B}$ is the smallest class of closed manifolds of positive dimension such that

- $\mathcal{B}$ contains each infranilmanifold [BK05], each closed manifold of $K \leq 0$ with a local Euclidean de Rham factor of positive dimension, and every circle bundle of type $(\mathrm{K})$;

- $\mathcal{B}$ is closed under products, disjoint unions, and products with any compact manifold of $K \leq 0$.

An orientable circle bundle has type $(\mathrm{K})$ if its base is a closed complex hyperbolic $n$-manifold whose holonomy representation lifts from $P U(n, 1)$ to $U(n, 1)$, and if the Euler class of the bundle equals $-m \frac{\omega}{4 \pi}$ for some nonzero integer $m$, where $\omega$ is the Kähler form of the base. For example, every nontrivial orientable circle bundle over a genus two orientable closed surface has type $(\mathrm{K})$, see [Bel13].

Question 18.4. Consider the class of closed manifolds $B$ to which Theorem 18.2 applies. Does the class contain every circle bundle over a closed manifold of $K<0$ ? Every closed infrasolvmanifold? Is the class closed under products? 


\section{TOPOLOGY OF KNOWN MANifolds OF BOUNDED NEGATIVE CURVATURE}

In dimensions $>3$ most (if not all) known examples of complete manifolds of $K \leq 0$ come from combining

- locally symmetric metrics of $K \leq 0$ arising from arithmetic or reflection groups,

- iterated and multiple warped products, building on the seminar work of Bishop-O'Neill on singly warped products of $K \leq 0$ [BO69].

Ontaneda's work Ont illustrates how the two methods combine: the block is an arithmetic real hyperbolic manifold with corners, and smoothing the metric involves sophisticated warped product considerations.

Prior examples of finite volume complete manifold of $K \leq 0$ that are not locally symmetric were typically produced by starting with a locally symmetric complete manifold of $K \leq 0$ and performing one of the following operations, which can be combined or iterated:

- doubles (Heintze, see [Sch91]) and twisted doubles Ont03,

- branched covers [MS80, GT87, FS90, Zhe96, ATM00, Der05, FO06,

- cusp closing Sch89, BK96, HS96, And06,

- cut and paste a tubular neighborhood of a totally geodesic submanifold of codimension 1, or dimensions 0 or 1, see [FJO07] for a survey,

- remove a family of totally geodesic submanifolds of codimension two, which results in an incomplete metric that in some cases can be modified to a complete metric of $K \leq 0$ [Fuj88, AS92, Buy93, Bel12a, Bel12b].

With few general results available, it makes sense to study topology of known examples in more detail. For the rest of the section let $M$ be an open connected complete finite volume manifold of $-1 \leq K<0$. The prior discussions gives

- (Section 6) $\pi_{1}(M)$ is acylindrically hyperbolic because $M$ has finite volume and $K<0$,

- (Theorem 15.4) $M$ is the interior of a compact manifold $N$, which is uniquely determined up to attaching an h-cobordism to the boundary.

- (Corollary 15.7) $\partial N$ has zero simplicial volume and Euler characteristic, and if $\partial N$ is orientable, $\partial N$ has zero Pontryagin numbers.

We would get a lot more information if $\pi_{1}(M)$ were hyperbolic relative to a collection of easy-to-understand peripheral subgroups (mainly because relatively hyperbolic groups inherit many properties from their peripheral subgroups, see below). Here is a prototypical example:

Example 19.1. If $M$ is negatively pinched (i.e $K$ is bounded between two negative constants), then $\pi_{1}(N)$ is hyperbolic relatively the fundamental groups of the components of $\partial N$ [Far98, Bow12] which are virtually nilpotent [BGS85]. 
One might expect that $\pi_{1}(M) \cong \pi_{1}(N)$ is always hyperbolic relative to the fundamental groups of components of $\partial N$. This idea runs into difficulties because $\partial N$ need not be $\pi_{1}$-incompressible Buy93, but when it works it does so to great effect, and become a major source of information about $\pi_{1}(M)$.

We refer to Osi06] for background on relatively hyperbolic groups; as a part of the definition we require that relatively hyperbolic groups are finitely generated and not virtually cyclic, and their peripheral subgroups are infinite and proper.

Theorem 19.2. (Belegradek) If $W$ is a compact aspherical manifold of dimension $\geq 3$ such that the components of $\partial W$ are aspherical and $\pi_{1}$-injectively embedded, and $\pi_{1}(W)$ is hyperbolic relatively to the fundamental groups of the components of $\partial W$, then

(a) if $\pi_{1}(W)$ splits nontrivially as amalgamated product or HNN extension over a subgroup $K$, then $K$ is acylindrically hyperbolic.

(b) $\pi_{1}(W)$ is co-Hopf, i.e. its injective endomorphisms are surjective.

(c) $\operatorname{Out}\left(\pi_{1}(W)\right)$ is finite if for every component $B$ of $\partial W$ the group $\pi_{1}(B)$ is either not relatively hyperbolic or has a relatively hyperbolic structure whose peripheral subgroups are not relatively hyperbolic.

Sketch of the proof. Mayer-Vietoris sequence in the group cohomology applied to the fundamental group of the double of $W$ along $\partial W$ can be used to show that $\pi_{1}(W)$ can only split over a non-elementary subgroup proving (a), which in turn implies (b)-(c) by results of Drutu-Sapir [DS08]. An alternative proof of (b) is immediate from the fact that $(W, \partial W)$ has positive simplicial norm [MY]. Details can be found in [Bel07].

Example 19.3. The fundamental group of a closed aspherical manifold with zero simplicial volume is not relatively hyperbolic (as noted in [BH13] this follows from [MY]). Thus if $W$ is as in Theorem 19.2 and $\operatorname{Int}(W)$ admits a complete metric finite volume of Ric $\geq-(n-1)$, then $\partial W$ has zero simplicial volume by Theorem 14.1, so $\operatorname{Out}\left(\pi_{1}(W)\right)$ is finite.

Proving relative hyperbolicity was the main goal of the author's work in Bel07, Bel12a, Bel12b, BH13, where it was accomplished in the following cases:

\section{Example 19.4.}

(1) If $R_{W, B}$ is from Section [18, then $\pi_{1}\left(R_{W, B}\right)$ is hyperbolic relative to the fundamental groups of components of $B$ [Bel07]. If $B$ is as in Theorem 18.3, then $R_{W, B}$ admits a complete finite volume metric of $-1 \leq K<0$.

(2) If $V$ is a closed manifold of $K<0$, and $S$ is an embedded, codimension two, compact, totally geodesic submanifold, then $\pi_{1}(V \backslash S)$ is hyperbolic relative to the fundamental groups of boundary components of a tubular neighborhood of $S$ in $V$ [BH13]. A finite volume complete metric of $-1 \leq K<0$ on $V \backslash S$ was 
constructed in Bel12a, Bel12b] when either $V$ is real hyperbolic, or $V$ and $S$ are complex hyperbolic.

(3) Let $V$ be a closed manifold of $K<0$, and $S$ be an immersed, codimension two, compact, totally geodesic submanifold whose preimage to the universal cover $\widetilde{V}$ of $V$ is normal in the sense of Allcock and is "sparse" in the sense that and two disjoint components are sufficiently separated. With these assumptions $\pi_{1}(V \backslash S)$ is hyperbolic relative to the fundamental group of the boundary components of a regular neighborhood of $S$ in $V$ [BH13. If $V$ is real hyperbolic, then $V \backslash S$ admits a complete finite volume metric of $-1 \leq K<0$ by [AS92].

Remark 19.5. A version of the claim in Example 19.4(2)-(3) holds when $V$ is an open complete finitely volume negatively pinched manifold.

Remark 19.6. Without the assumptions that the preimage of $S$ to $\widetilde{V}$ is normal and "sparse", it seems unlikely that $\pi_{1}(V \backslash S)$ in Example 19.4(3) is hyperbolic relative to some easy-to-understand peripheral subgroups, so we ask:

Question 19.7. Let $V$ be a finite volume complete negatively pinched manifold, and $S$ be an immersed, codimension two, compact, totally geodesic submanifold. Is $\pi_{1}(V \backslash S)$ acylindrically hyperbolic?

If $G$ is a finitely generated group that is hyperbolic relatively to a finite family of peripheral subgroups, then $G$ inherits the following properties of its peripheral subgroups:

(1) solvability of the word problem [Far98, Osi06] (which is a litmus test for decency of a group).

(2) solvability of conjugacy problem [Bum04].

(3) being fully residually hyperbolic Osi07, GM08; here given a class of groups $\mathcal{C}$, a group $G$ is fully residually $\mathcal{C}$ if any finite subset of $G$ can be mapped injectively by a homomorphism of $G$ onto a group in $\mathcal{C}$.

(4) being biautomatic Reb01.

(5) finiteness of asymptotic dimension Osi05

(6) rapid decay property DS05]

(7) Tits alternative: a subgroup of a relatively hyperbolic group that does not contain a non-abelian free subgroup is elementary, i.e. virtually- $\mathbb{Z}$, finite, or contained in a peripheral subgroup [Tuk94].

Example 19.8. If $V$ and $S$ are as in Example 19.4(2), then the peripheral subgroups in the relatively hyperbolic groups structure on $\pi_{1}(V \backslash S)$ are the fundamental groups of circle bundles over components of $S$. The peripheral subgroups have solvable word and conjugacy problems, have finite asymptotic dimension and rapid decay property, are biautomatic, residually hyperbolic, and their non-virtually-abelian groups contain free nonabelian subgroups, see [Bel12a, Bel12b]. Hence all these properties are inherited by $\pi_{1}(V \backslash S)$. 
The fact that most real hyperbolic manifolds are a-Kähler Gro93 was used in Bel07] to show

Theorem 19.9. $R_{W, B}$ is not homeomorphic to an open subset of a Kähler manifold of real dimension $\geq 4$.

In fact, "not homeomorphic" can be replaced with "not proper homotopy equivalent" under a mild assumption on the strict hyperbolization block [Bel07].

\section{ZOO OF FINITE VOLUME RANK ONE MANIFOLDS}

In this section we discuss examples and structure of connected open complete finite volume manifolds of $K \leq 0$ and rank one, with a particular focus on manifolds of $K<0$.

Theorem 20.1. If $V$ is a complete finite volume manifold of rank one, then $\pi_{1}(V)$ is acylindically hyperbolic.

Proof. The universal cover $\widetilde{V}$ has rank one, and Ballmann Bal95 proved that a lattice in the isometry group of a rank one Hadamard manifold contains a rank one element that lies in a noncyclic free subgroup, so Sisto's Theorem 6.1 applies.

Question 20.2. Does every complete rank one manifold with $K \leq 0$ and Inj Rad $\rightarrow 0$ have acylindically hyperbolic fundamental group?

Kapovitch-Wilking's version of the Margulis lemma only calls for $K \geq-1$ on a ball of radius 1, and that curvature bound can always be achieved by rescaling. Since acylindically hyperbolic groups are not virtually nilpotent, Theorem 14.6 immediately implies:

Corollary 20.3. If $\varepsilon$ is the constant in Theorem 14.6, then a finite volume complete rank one manifold $M$ contains no point $p$ such that $K \geq-1$ on $B_{p}(1)$ and the inclusion $B_{p}(\varepsilon) \hookrightarrow M$ is $\pi_{1}$-surjective.

Thus if $\pi_{1}(M)$ is "concentrated" on an $\varepsilon$-ball, then $K$ blows up near that ball.

Known examples of finite volume complete manifolds of $K<0$ that admit no finite volume metric of $-1 \leq K \leq 0$ are based on Theorem 14.1 that a compact boundary component of a manifold whose interior has a complete metric of Ric $\geq-(n-1)$ has zero simplicial volume. Indeed, Nguyen Phan $\mathrm{NPb}$ proved

Theorem 20.4. (Nguyen Phan) In each dimension $\geq 3$ there exists a finite volume complete manifold of $K<0$ that is the interior of a compact manifold whose boundary admits a real hyperbolic metric. 
Recall that a closed real hyperbolic manifold has a positive simplicial volume. The proof of the above theorem is by explicit construction; alternatively, it follows from Ontaneda's Theorem 18.2 by inserting in the cusp the warped product $\mathbb{R} \times_{e^{r}} B$ where $B$ is any closed manifold of $K \leq 0$ with nonzero simplicial volume. In fact, this argument proves:

Theorem 20.5. (Ontaneda) If a closed manifold of $K \leq 0$ is diffeomorphic to the boundary of a compact manifold, then it is diffeomorphic to the boundary of a compact manifold whose interior admits a complete finite volume metric of $K \leq-1$.

Example 20.6. There are closed manifolds of $K \leq 0$ that are not homeomorphic to the boundaries of compact manifolds. One source of such examples is evenness of the Euler characteristic of the boundary of a compact manifold Dol95, Corollary VIII.8.8]. Examples of closed manifold of $K \leq 0$ with odd Euler characteristic include suitable closed non-orientable hyperbolic surfaces, Mumford's complex hyperbolic surface of Euler characteristic 3 HP96, Proposition2.2], or their products.

Iterated gluing along totally geodesic boundaries sometimes yields finite volume $M$ with infinitely generated fundamental group. This phenomenon was discovered by Gromov [BGS85, Chapter 11] who produced such 3-dimensional graph manifolds with $K \in[-1,0]$ and no local Euclidean de Rham factor. Other examples with infinitely generated fundamental groups, due to Nguyen Phan $\mathrm{NPb}$, are finite volume manifolds with $K \leq-1$ and infinitely many ends, appearing in all dimensions $\geq 2$.

A related idea of Nguyen Phan [NPb] uses infinite cyclic covers of closed manifolds of $K<0$ to produce two-ended finite volume manifolds of $K<0$ :

Theorem 20.7. (Nguyen Phan) If $\widehat{L}$ is an infinite cyclic cover of a closed manifold $L$ of $K<0$ of dimension $l \geq 3$, then $\widehat{L}$ admits a complete finite volume metric metric of $K<0$.

Here $\widehat{L} \rightarrow L$ is the covering that corresponds to the kernel of any epimorphism $\pi_{1}(L) \rightarrow \mathbb{Z}$. A most famous example is when $\widehat{L}$ corresponds to the fiber group in a closed hyperbolic 3 -manifolds that fibers over a circle.

Example 20.8. (of $\widehat{L}$ with infinitely generated fundamental group) Suppose that $\pi_{1}(L)$ surjects onto a noncyclic free group $F_{r}$. The kernel of any epimorphism $F_{r} \rightarrow \mathbb{Z}$ is infinitely generated, so hence so is the kernel of the composite $\pi_{1}(L) \rightarrow F_{r} \rightarrow \mathbb{Z}$.

Question 20.9. Is $\pi_{1}(\widehat{L})$ always infinitely generated when $\operatorname{dim}(\widehat{L})>3$ ? 
Remark 20.10. If $\operatorname{dim}(\widehat{L}) \geq 6$ and $\widehat{L}$ is homotopy equivalent to a finite cell complex (or more generally is finitely dominated), then $L$ (smoothly) fibers over a circle, and the fiber is a closed aspherical manifold whose inclusion into $L$ corresponds homotopically to the covering $\widehat{L} \rightarrow L$. Indeed, Siebenmann's version [Sie70] of Farrell's fibering obstruction lies in the group $\mathrm{Wh}\left(\pi_{1}(L)\right)$, which is zero by FJ86.

Remark 20.11. Another restriction on $\pi_{1}(\widehat{L})$ is that its outer automorphism group is infinite (as easily follows from the fact that $\pi_{1}(\widehat{L})$ has trivial centralizer in $\left.\pi_{1}(L)\right)$. Combining with the previous remark we see that if $\operatorname{dim}(\widehat{L}) \geq 6$ and $\widehat{L}$ is finitely dominated, then $\widehat{L}$ is homotopy equivalent to a closed aspherical manifold of dimension $\geq 5$ whose fundamental group has infinite outer automorphism group and embeds into the hyperbolic group $\pi_{1}(L)$; it seems such a closed aspherical manifold cannot exist, so we ask:

Question 20.12. Can $\widehat{L}$ ever be finitely dominated when $\operatorname{dim}(\widehat{L})>3$ ?

Example 20.13. In dimension three Theorem 20.7 gives a finite volume metric of $K<0$ on the product of a closed hyperbolic surface with $\mathbb{R}$, and interestingly, the metric can be chosen so that the corresponding nonuniform lattice contains no parabolics, see $[\mathrm{NPb}]$.

Question 20.14. Is there a nonuniform lattice in the isometry group of a Hadamard manifold of dimension $>3$ that contains no parabolics?

Borel-Serre [BS73] compute the $\mathbb{Q}$-rank of a finite volume complete locally symmetric $n$-manifold $V$ as $n-\operatorname{cd}\left(\pi_{1}(V)\right)$, where cd denotes the cohomological dimension; alternatively, $\mathbb{Q}$-rank equals the dimension of an asymptotic cone of $V$ Hat96], cf. [JM02, and $\mathbb{Q}$-rank can also be defined in terms of flats in $V$ Mor. If $V$ has rank one (i.e. contains a rank one geodesic), then the $\mathbb{Q}$ rank of $V$ equals 1 . One wonders whether any of these relations between cd, asymptotic cone, and absence of 2-dimensional flats extend to finite volume complete manifolds of rank one.

Question 20.15. Is the asymptotic cone of a complete finite volume rank one manifold of $K \leq 0$ always a tree?

Question 20.16. What values does cd take on the fundamental groups of open complete finite volume $n$-manifolds of $K \leq-1$, rank one, or $K<0$ ? Is $\mathrm{cd}$ always equal $n-1$ ?

To better understand the above question let us relate bounds on cd with the fundamental group at infinity of an open aspherical $n$-manifold $M$. First note that if $\operatorname{cd}\left(\pi_{1}(M)\right) \leq n-2$, then $M$ is one-ended [Sie69, Proposition 1.2] but the converse fails (think of the open Möbius band). In the simplest case when $M$ is the interior of a compact manifold, we get the following clean statement: 
Proposition 20.17. Let $N$ be a compact aspherical $n$-manifold with boundary. Then $\operatorname{cd}\left(\pi_{1}(M)\right) \leq n-2$ if and only if $\partial N$ is connected and the inclusion $\partial N \hookrightarrow N$ is $\pi_{1}$-surjective.

Proof. Clearly $\operatorname{cd}\left(\pi_{1}(M)\right) \leq n-1$. Now Poincaré-Lefschetz duality in the universal cover [Bro94, Corollary VIII.8.3] implies that $\operatorname{cd}\left(\pi_{1}(M)\right)=n-1$ if and only if the boundary of the universal cover of $N$ is not connected. The latter is equivalent to "either $\partial N$ is not connected or $\partial N \hookrightarrow N$ is not $\pi_{1}$-surjective" by elementary covering space considerations.

Question 20.16 should be compared with Nguyen Phan's Example 20.13 of a finite volume manifold of $K<0$ that is quite small homologically, and perhaps there are even smaller examples. We finish with the following tantalizing

Question 20.18. Given $n>2$, does the interior of the $n$-dimensional handlebody admit a complete finite volume metric of $K \leq 0$ ?

Example 20.19. The interior of an odd-dimensional handlebody admits no complete finite volume metric of $-1 \leq K \leq 0$. (The boundary of an odddimensional handlebody with $g$ handles has Euler characteristic $2-2 g$ while under our geometric assumptions the Euler characteristic vanishes by Theorem 15.7 and $g \neq 1$ because $\mathbb{Z}$ is not acylindrically hyperbolic).

\section{REFERENCES}

[AB98] S. Adams and W. Ballmann, Amenable isometry groups of Hadamard spaces, Math. Ann. 312 (1998), no. 1, 183-195.

$\left[\mathrm{ABJ}^{+}\right.$09] G. Arzhantseva, M. R. Bridson, T. Januszkiewicz, I. J. Leary, A. Minasyan, and J. Świątkowski, Infinite groups with fixed point properties, Geom. Topol. 13 (2009), no. 3, 1229-1263.

[AFW] M. Aschenbrenner, S. Friedl, and H. Wilton, 3-manifold groups, arXiv:1205.0202.

[Ago] I. Agol, Tameness of hyperbolic 3-manifolds, arXiv:math/0405568.

[Ago13] _ The virtual Haken conjecture, Doc. Math. 18 (2013), 1045-1087, With an appendix by Agol, D. Groves, and J. Manning.

[And87] M. T. Anderson, Metrics of negative curvature on vector bundles, Proc. Amer. Math. Soc. 99 (1987), no. 2, 357-363.

[And06] _ Dehn filling and Einstein metrics in higher dimensions, J. Differential Geom. 73 (2006), no. 2, 219-261.

[AS82] R. C. Alperin and P. B. Shalen, Linear groups of finite cohomological dimension, Invent. Math. 66 (1982), no. 1, 89-98.

[AS92] U. Abresch and V. Schroeder, Graph manifolds, ends of negatively curved spaces and the hyperbolic 120 -cell space, J. Differential Geom. 35 (1992), no. 2, 299-336.

[ATM00] S. I. Ardanza-Trevijano Moras, Exotic smooth structures on negatively curved manifolds that are not of the homotopy type of a locally symmetric space, ProQuest LLC, Ann Arbor, MI, 2000, Thesis (Ph.D.)-SUNY at Binghamton.

[Bal95] W. Ballmann, Lectures on spaces of nonpositive curvature, DMV Seminar, vol. 25, Birkhäuser Verlag, Basel, 1995, With an appendix by Misha Brin. 
[Bav91] C. Bavard, Longueur stable des commutateurs, Enseign. Math. (2) 37 (1991), no. 12, 109-150.

[BB08] W. Ballmann and S. Buyalo, Periodic rank one geodesics in Hadamard spaces, Geometric and probabilistic structures in dynamics, Contemp. Math., vol. 469, Amer. Math. Soc., Providence, RI, 2008, pp. 19-27.

[Bel] I. Belegradek, Obstructions to nonpositive curvature for open manifolds, arXiv:1208.5220v1, to appear in Proc. Lond. Math. Soc.

[Bel01] _ Pinching, Pontrjagin classes, and negatively curved vector bundles, Invent. Math. 144 (2001), no. 2, 353-379.

[Bel07] - Aspherical manifolds with relatively hyperbolic fundamental groups, Geom. Dedicata 129 (2007), 119-144.

[Bel12a] C Complex hyperbolic hyperplane complements, Math. Ann. 353 (2012), no. $2,545-579$.

[Bel12b] Rigidity and relative hyperbolicity of real hyperbolic hyperplane complements, Pure Appl. Math. Q. 8 (2012), no. 1, 15-51.

[Bel13] 439-449.

[BF02a] M. Bestvina and M. Feighn, Proper actions of lattices on contractible manifolds, Invent. Math. 150 (2002), no. 2, 237-256.

[BF02b] M. Bestvina and K. Fujiwara, Bounded cohomology of subgroups of mapping class groups, Geom. Topol. 6 (2002), 69-89.

[BF09] _ A characterization of higher rank symmetric spaces via bounded cohomology, Geom. Funct. Anal. 19 (2009), no. 1, 11-40.

[BFL14] A. Bartels, F. T. Farrell, and W. Lück, The Farrell-Jones Conjecture for cocompact lattices in virtually connected Lie groups, J. Amer. Math. Soc. 27 (2014), no. 2, 339-388.

[BGS85] W. Ballmann, M. Gromov, and V. Schroeder, Manifolds of nonpositive curvature, Progress in Mathematics, vol. 61, Birkhäuser Boston Inc., Boston, MA, 1985.

[BH99] M. R. Bridson and A. Haefliger, Metric spaces of non-positive curvature, Grundlehren der Mathematischen Wissenschaften [Fundamental Principles of Mathematical Sciences], vol. 319, Springer-Verlag, Berlin, 1999.

[BH13] I. Belegradek and G. C. Hruska, Hyperplane arrangements in negatively curved manifolds and relative hyperbolicity, Groups Geom. Dyn. 7 (2013), no. 1, 13-38.

[Bie81] R. Bieri, Homological dimension of discrete groups, second ed., Queen Mary College Mathematical Notes, Queen Mary College Department of Pure Mathematics, London, 1981.

[BJ05] A. Borel and L. Ji, Compactifications of symmetric and locally symmetric spaces, Lie theory, Progr. Math., vol. 229, Birkhäuser Boston, Boston, MA, 2005, pp. 69137.

[BK95] S. V. Buyalo and V. L. Kobel'skii, Geometrization of graph-manifolds. II. Isometric geometrization, Algebra i Analiz 7 (1995), no. 3, 96-117.

[BK96] Cusp closing of hyperbolic manifolds, Geom. Dedicata 59 (1996), no. 2, $147-156$.

[BK05] I. Belegradek and V. Kapovitch, Pinching estimates for negatively curved manifolds with nilpotent fundamental groups, Geom. Funct. Anal. 15 (2005), no. 5, 929-938.

[BK06] Classification of negatively pinched manifolds with amenable fundamental groups, Acta Math. 196 (2006), no. 2, 229-260.

[BKK02] M. Bestvina, M. Kapovich, and B. Kleiner, Van Kampen's embedding obstruction for discrete groups, Invent. Math. 150 (2002), no. 2, 219-235. 
[BL05] A. Balser and A. Lytchak, Centers of convex subsets of buildings, Ann. Global Anal. Geom. 28 (2005), no. 2, 201-209.

[BL12] A. Bartels and W. Lück, The Borel conjecture for hyperbolic and CAT(0)-groups, Ann. of Math. (2) 175 (2012), no. 2, 631-689.

[BM02] M. Burger and N. Monod, Continuous bounded cohomology and applications to rigidity theory, Geom. Funct. Anal. 12 (2002), no. 2, 219-280.

[BNP] I. Belegradek and T. T. Nguyễn Phan, Non-aspherical ends and nonpositive curvature, arXiv:1212.3303.

[BO69] R. L. Bishop and B. O'Neill, Manifolds of negative curvature, Trans. Amer. Math. Soc. 145 (1969), 1-49.

[Bow10] B. H. Bowditch, Notes on tameness, Enseign. Math. (2) 56 (2010), no. 3-4, 229 285.

[Bow12] _ Relatively hyperbolic groups, Internat. J. Algebra Comput. 22 (2012), no. 3, 1250016, 66 .

[Bri01] M. R. Bridson, On the subgroups of semihyperbolic groups, Essays on geometry and related topics, Vol. 1, 2, Monogr. Enseign. Math., vol. 38, Enseignement Math., Geneva, 2001, pp. 85-111.

[Bro94] K. S. Brown, Cohomology of groups, Graduate Texts in Mathematics, vol. 87, Springer-Verlag, New York, 1994, Corrected reprint of the 1982 original.

[BS73] A. Borel and J.-P. Serre, Corners and arithmetic groups, Comment. Math. Helv. 48 (1973), 436-491, Avec un appendice: Arrondissement des variétés à coins, par A. Douady et L. Hérault.

[BS87a] F. Bonahon and L. C. Siebenmann, The characteristic toric splitting of irreducible compact 3 -orbifolds, Math. Ann. 278 (1987), no. 1-4, 441-479.

[BS87b] M. Burger and V. Schroeder, Amenable groups and stabilizers of measures on the boundary of a Hadamard manifold, Math. Ann. 276 (1987), no. 3, 505-514.

[BS04] S. V. Buyalo and P. V. Svetlov, Topological and geometric properties of graph manifolds, Algebra i Analiz 16 (2004), no. 2, 3-68.

[Bum04] I. Bumagin, The conjugacy problem for relatively hyperbolic groups, Algebr. Geom. Topol. 4 (2004), 1013-1040.

[Buy93] S. V. Buyalo, An example of a four-dimensional manifold of negative curvature, Algebra i Analiz 5 (1993), no. 1, 193-199.

[Can08] R. D. Canary, Marden's tameness conjecture: history and applications, Geometry, analysis and topology of discrete groups, Adv. Lect. Math. (ALM), vol. 6, Int. Press, Somerville, MA, 2008, pp. 137-162.

[CD95] R. M. Charney and M. W. Davis, Strict hyperbolization, Topology 34 (1995), no. 2, 329-350.

[CF10] P.-E. Caprace and K. Fujiwara, Rank-one isometries of buildings and quasimorphisms of Kac-Moody groups, Geom. Funct. Anal. 19 (2010), no. 5, 1296-1319.

[CG85] J. Cheeger and M. Gromov, Bounds on the von Neumann dimension of $L^{2}$ cohomology and the Gauss-Bonnet theorem for open manifolds, J. Differential Geom. 21 (1985), no. 1, 1-34.

[CG91] C Chopping Riemannian manifolds, Differential geometry, Pitman Monogr. Surveys Pure Appl. Math., vol. 52, Longman Sci. Tech., Harlow, 1991, pp. 85-94.

[CG06] D. Calegari and D. Gabai, Shrinkwrapping and the taming of hyperbolic 3manifolds, J. Amer. Math. Soc. 19 (2006), no. 2, 385-446.

[CL10] P.-E. Caprace and A. Lytchak, At infinity of finite-dimensional CAT(0) spaces, Math. Ann. 346 (2010), no. 1, 1-21. 
[CM04] R. D. Canary and D. McCullough, Homotopy equivalences of 3-manifolds and deformation theory of Kleinian groups, Mem. Amer. Math. Soc. 172 (2004), no. 812, xii +218 .

[CM09a] P.-E. Caprace and N. Monod, Isometry groups of non-positively curved spaces: discrete subgroups, J. Topol. 2 (2009), no. 4, 701-746.

[CM09b] _ Isometry groups of non-positively curved spaces: structure theory, J. Topol. 2 (2009), no. 4, 661-700.

[Daf97] M. Dafermos, Exhaustions of complete manifolds of bounded curvature, Tsing Hua lectures on geometry \& analysis (Hsinchu, 1990-1991), Int. Press, Cambridge, MA, 1997, pp. 319-322.

[Dav08] M. W. Davis, The geometry and topology of Coxeter groups, London Mathematical Society Monographs Series, vol. 32, Princeton University Press, Princeton, NJ, 2008.

[Der05] M. Deraux, A negatively curved Kähler threefold not covered by the ball, Invent. Math. 160 (2005), no. 3, 501-525.

[Des06] Z. Despotovic, Action dimension of mapping class groups, ProQuest LLC, Ann Arbor, MI, 2006, Thesis (Ph.D.)-The University of Utah.

[DGO] F. Dahmani, V. Guirardel, and D. Osin, Hyperbolically embedded subgroups and rotating families in groups acting on hyperbolic spaces, http://arXiv:1111.7048v3.

[DJ91] M. W. Davis and T. Januszkiewicz, Hyperbolization of polyhedra, J. Differential Geom. 34 (1991), no. 2, 347-388.

[DJW01] M. W. Davis, T. Januszkiewicz, and S. Weinberger, Relative hyperbolization and aspherical bordisms: an addendum to "Hyperbolization of polyhedra", J. Differential Geom. 58 (2001), no. 3, 535-541.

[Dol95] A. Dold, Lectures on algebraic topology, Classics in Mathematics, Springer-Verlag, Berlin, 1995, Reprint of the 1972 edition.

[DS05] C. Druţu and M. Sapir, Relatively hyperbolic groups with rapid decay property, Int. Math. Res. Not. (2005), no. 19, 1181-1194.

[DS08] C. Druţu and M. V. Sapir, Groups acting on tree-graded spaces and splittings of relatively hyperbolic groups, Adv. Math. 217 (2008), no. 3, 1313-1367.

[Duc] B. Duchesne, Superrigidity in infinite dimension and finite rank via harmonic maps, arXiv:1206.1964v1.

[Ebe79] P. Eberlein, Surfaces of nonpositive curvature, Mem. Amer. Math. Soc. 20 (1979), no. $218, \mathrm{x}+90$.

[Ebe80] L Lattices in spaces of nonpositive curvature, Ann. of Math. (2) 111 (1980), no. $3,435-476$.

[Ebe96] P. B. Eberlein, Geometry of nonpositively curved manifolds, Chicago Lectures in Mathematics, University of Chicago Press, Chicago, IL, 1996.

[EO73] P. Eberlein and B. O'Neill, Visibility manifolds, Pacific J. Math. 46 (1973), 45-109.

[Eym72] P. Eymard, Moyennes invariantes et représentations unitaires, Lecture Notes in Mathematics, Vol. 300, Springer-Verlag, Berlin, 1972.

[Far98] B. Farb, Relatively hyperbolic groups, Geom. Funct. Anal. 8 (1998), no. 5, 810-840.

[FG07] M. H. Freedman and D. Gabai, Covering a nontaming knot by the unlink, Algebr. Geom. Topol. 7 (2007), 1561-1578.

[FJ86] F. T. Farrell and L. E. Jones, $K$-theory and dynamics. I, Ann. of Math. (2) 124 (1986), no. 3, 531-569.

[FJO07] F. T. Farrell, L. E. Jones, and P. Ontaneda, Negative curvature and exotic topology, Surveys in differential geometry. Vol. XI, Surv. Differ. Geom., vol. 11, Int. Press, Somerville, MA, 2007, pp. 329-347. 
[FNS06] K. Fujiwara, K. Nagano, and T. Shioya, Fixed point sets of parabolic isometries of CAT(0)-spaces, Comment. Math. Helv. 81 (2006), no. 2, 305-335.

[FO06] F. T. Farrell and P. Ontaneda, Branched covers of hyperbolic manifolds and harmonic maps, Comm. Anal. Geom. 14 (2006), no. 2, 249-268.

[FS90] S. Fornari and V. Schroeder, Ramified coverings with nonpositive curvature, Math. Z. 203 (1990), no. 1, 123-128.

[Fuj88] K. Fujiwara, A construction of negatively curved manifolds, Proc. Japan Acad. Ser. A Math. Sci. 64 (1988), no. 9, 352-355.

[Fuk84] K. Fukaya, A finiteness theorem for negatively curved manifolds, J. Differential Geom. 20 (1984), no. 2, 497-521.

[Fur11] A. Furman, A survey of measured group theory, Geometry, rigidity, and group actions, Chicago Lectures in Math., Univ. Chicago Press, Chicago, IL, 2011, pp. 296374 .

[FY92] K. Fukaya and T. Yamaguchi, The fundamental groups of almost non-negatively curved manifolds, Ann. of Math. (2) 136 (1992), no. 2, 253-333.

[Gel04] T. Gelander, Homotopy type and volume of locally symmetric manifolds, Duke Math. J. 124 (2004), no. 3, 459-515.

[Gel11] _ Volume versus rank of lattices, J. Reine Angew. Math. 661 (2011), 237248.

[GM08] D. Groves and J. F. Manning, Dehn filling in relatively hyperbolic groups, Israel J. Math. 168 (2008), 317-429.

[Gol71] M. E. Goldman, An algebraic classification of noncompact 2 -manifolds, Trans. Amer. Math. Soc. 156 (1971), 241-258.

[Gro78] M. Gromov, Manifolds of negative curvature, J. Differential Geom. 13 (1978), no. 2, 223-230.

[Gro82] ㄴ Volume and bounded cohomology, Inst. Hautes Études Sci. Publ. Math. (1982), no. 56, 5-99 (1983).

[Gro87] Hyperbolic groups, Essays in group theory, Math. Sci. Res. Inst. Publ., vol. 8, Springer, New York, 1987, pp. 75-263.

[Gro93] - Asymptotic invariants of infinite groups, Geometric group theory, Vol. 2 (Sussex, 1991), London Math. Soc. Lecture Note Ser., vol. 182, Cambridge Univ. Press, Cambridge, 1993, pp. 1-295.

[Gro03] , Random walk in random groups, Geom. Funct. Anal. 13 (2003), no. 1, 73-146.

[GT87] M. Gromov and W. Thurston, Pinching constants for hyperbolic manifolds, Invent. Math. 89 (1987), no. 1, 1-12.

[Gui07] C. R. Guilbault, Products of open manifolds with $\mathbb{R}$, Fund. Math. 197 (2007), 197-214.

[GW71] D. Gromoll and J. A. Wolf, Some relations between the metric structure and the algebraic structure of the fundamental group in manifolds of nonpositive curvature, Bull. Amer. Math. Soc. 77 (1971), 545-552.

[Ham08] U. Hamenstädt, Bounded cohomology and isometry groups of hyperbolic spaces, J. Eur. Math. Soc. (JEMS) 10 (2008), no. 2, 315-349.

[Ham09a] Isometry groups of proper hyperbolic spaces, Geom. Funct. Anal. 19 (2009), no. $1,170-205$.

[Ham09b] _ Rank-one isometries of proper CAT(0)-spaces, Discrete groups and geometric structures, Contemp. Math., vol. 501, Amer. Math. Soc., Providence, RI, 2009, pp. 43-59. 
[Ham12] Isometry groups of proper CAT(0)-spaces of rank one, Groups Geom. Dyn. 6 (2012), no. 3, 579-618.

[Hat] A. Hatcher, Basic 3-manifold topology, www.math.cornell.edu/ hatcher/.

[Hat96] T. Hattori, Asymptotic geometry of arithmetic quotients of symmetric spaces, Math. Z. 222 (1996), no. 2, 247-277.

[HO13] M. Hull and D. Osin, Induced quasicocycles on groups with hyperbolically embedded subgroups, Algebr. Geom. Topol. 13 (2013), no. 5, 2635-2665.

[HP96] S. Hersonsky and F. Paulin, On the volumes of complex hyperbolic manifolds, Duke Math. J. 84 (1996), no. 3, 719-737.

[HS96] C. Hummel and V. Schroeder, Cusp closing in rank one symmetric spaces, Invent. Math. 123 (1996), no. 2, 283-307.

[IKN12] H. Izeki, T. Kondo, and S. Nayatani, $N$-step energy of maps and the fixed-point property of random groups, Groups Geom. Dyn. 6 (2012), no. 4, 701-736.

[IN05] H. Izeki and S. Nayatani, Combinatorial harmonic maps and discrete-group actions on Hadamard spaces, Geom. Dedicata 114 (2005), 147-188.

[IN10] - An approach to superrigidity and fixed-point theorems via harmonic maps, Selected papers on analysis and differential equations, Amer. Math. Soc. Transl. Ser. 2, vol. 230, Amer. Math. Soc., Providence, RI, 2010, pp. 135-160.

[JM02] L. Ji and R. MacPherson, Geometry of compactifications of locally symmetric spaces, Ann. Inst. Fourier (Grenoble) 52 (2002), no. 2, 457-559.

[Joh72] B. E. Johnson, Cohomology in Banach algebras, American Mathematical Society, Providence, R.I., 1972, Memoirs of the American Mathematical Society, No. 127.

[Kap01] M. Kapovich, Hyperbolic manifolds and discrete groups, Progress in Mathematics, vol. 183, Birkhäuser Boston Inc., Boston, MA, 2001

[Ker23] B. Kerékjártó, Vorlesungen über Topologie, I, Springer, Berlin, 1923.

[KL96] M. Kapovich and B. Leeb, Actions of discrete groups on nonpositively curved spaces, Math. Ann. 306 (1996), no. 2, 341-352.

[Kle99] B. Kleiner, Integrating infinitesimal flats in Hadamard manifolds, preprint, 1999.

[KN04] A. Karlsson and G. A. Noskov, Some groups having only elementary actions on metric spaces with hyperbolic boundaries, Geom. Dedicata 104 (2004), 119-137.

[KPT10] V. Kapovitch, A. Petrunin, and W. Tuschmann, Nilpotency, almost nonnegative curvature, and the gradient flow on Alexandrov spaces, Ann. of Math. (2) 171 (2010), no. 1, 343-373.

[KW] V. Kapovitch and B. Wilking, Structure of fundamental groups of manifolds with Ricci curvature bounded below, arXiv:1105.5955

[Lee95] B. Leeb, 3-manifolds with(out) metrics of nonpositive curvature, Invent. Math. 122 (1995), no. 2, 277-289.

[Leu95] E. Leuzinger, An exhaustion of locally symmetric spaces by compact submanifolds with corners, Invent. Math. 121 (1995), no. 2, 389-410.

[Leu04] On polyhedral retracts and compactifications of locally symmetric spaces, Differential Geom. Appl. 20 (2004), no. 3, 293-318.

[Liu13] Yi Liu, Virtual cubulation of nonpositively curved graph manifolds, J. Topol. 6 (2013), no. 4, 793-822.

[Lüc10] W. Lück, Survey on aspherical manifolds, European Congress of Mathematics, Eur. Math. Soc., Zürich, 2010, pp. 53-82.

[LY72] H. B. Lawson, Jr. and S. T. Yau, Compact manifolds of nonpositive curvature, J. Differential Geometry 7 (1972), 211-228. 
[Mar91] G. A. Margulis, Discrete subgroups of semisimple Lie groups, Ergebnisse der Mathematik und ihrer Grenzgebiete (3) [Results in Mathematics and Related Areas (3)], vol. 17, Springer-Verlag, Berlin, 1991.

[MMS04] I. Mineyev, N. Monod, and Y. Shalom, Ideal bicombings for hyperbolic groups and applications, Topology 43 (2004), no. 6, 1319-1344.

[MO] A. Minasyan and D. Osin, Acylindrically hyperbolic groups acting on trees, in preparation.

[Mon06a] N. Monod, An invitation to bounded cohomology, International Congress of Mathematicians. Vol. II, Eur. Math. Soc., Zürich, 2006, pp. 1183-1211.

[Mon06b] _ Superrigidity for irreducible lattices and geometric splitting, J. Amer. Math. Soc. 19 (2006), no. 4, 781-814.

[Moo09] S. Moon, Amenable actions of discrete groups, Ph.D. thesis, Universite de Neuchatel, http://doc.rero.ch/record/18088, 2009.

[Mor] D. Morris, Introduction to arithmetic groups, arXiv:math/0106063.

[MP03] N. Monod and S. Popa, On co-amenability for groups and von Neumann algebras, C. R. Math. Acad. Sci. Soc. R. Can. 25 (2003), no. 3, 82-87.

[MS80] G. D. Mostow and Y. T. Siu, A compact Kähler surface of negative curvature not covered by the ball, Ann. of Math. (2) 112 (1980), no. 2, 321-360.

[MS04] N. Monod and Y. Shalom, Cocycle superrigidity and bounded cohomology for negatively curved spaces, J. Differential Geom. 67 (2004), no. 3, 395-455.

[MS06] - Orbit equivalence rigidity and bounded cohomology, Ann. of Math. (2) 164 (2006), no. 3, 825-878.

[MY] I. Mineyev and A. Yaman, Relative hyperbolicity and bounded cohomology, www.math.uiuc.edu/ mineyev/math/

[Nos90] G. A. Noskov, Bounded cohomology of discrete groups with coefficients, Algebra i Analiz 2 (1990), no. 5, 146-164.

[NPa] T. T. Nguyễn Phan, Nil happens. What about Sol?, arXiv:1207.1734.

[NPb] _ On finite volume, negatively curved manifolds, arXiv:1110.4087.

[NP13] _ Nonpositively curved manifolds containing a prescribed nonpositively curved hypersurface, Topology Proc. 42 (2013), 39-41.

[NS11] A. Naor and L. Silberman, Poincaré inequalities, embeddings, and wild groups, Compos. Math. 147 (2011), no. 5, 1546-1572.

[OH07] A. Ould Houcine, Embeddings in finitely presented groups which preserve the center, J. Algebra 307 (2007), no. 1, 1-23.

[Ont] P. Ontaneda, Pinched smooth hyperbolization, arXiv:1110.6374v1.

[Ont03] The double of a hyperbolic manifold and non-positively curved exotic PL structures, Trans. Amer. Math. Soc. 355 (2003), no. 3, 935-965 (electronic).

[Osi] D. V. Osin, Acylindrically hyperbolic groups, arXiv:1304.1246v1.

[Osi05] Asymptotic dimension of relatively hyperbolic groups, Int. Math. Res. Not. (2005), no. 35, 2143-2161.

[Osi06] , Relatively hyperbolic groups: intrinsic geometry, algebraic properties, and algorithmic problems, Mem. Amer. Math. Soc. 179 (2006), no. 843, vi+100.

[Osi07] , Peripheral fillings of relatively hyperbolic groups, Invent. Math. 167 (2007), no. 2, 295-326.

[Reb01] D. Y. Rebbechi, Algorithmic properties of relatively hyperbolic groups, Ph.D. thesis, Rutgers Newark arXiv:math/0302245v1, 2001.

[Ric63] I. Richards, On the classification of noncompact surfaces, Trans. Amer. Math. Soc. 106 (1963), 259-269. 
[Sap97] L. Saper, Tilings and finite energy retractions of locally symmetric spaces, Comment. Math. Helv. 72 (1997), no. 2, 167-202.

[Sch85] V. Schroeder, A splitting theorem for spaces of nonpositive curvature, Invent. Math. 79 (1985), no. 2, 323-327.

[Sch89] _ A cusp closing theorem, Proc. Amer. Math. Soc. 106 (1989), no. 3, 797802.

[Sch91] Analytic manifolds of nonpositive curvature with higher rank subspaces, Arch. Math. (Basel) 56 (1991), no. 1, 81-85.

[Sie] L. C. Siebenmann, Classifcation des 2-variéetés ouvertes, preprint, 2008.

[Sie69] _ On detecting open collars, Trans. Amer. Math. Soc. 142 (1969), 201-227.

[Sie70] A total Whitehead torsion obstruction to fibering over the circle, Comment. Math. Helv. 45 (1970), 1-48.

[Sis] A. Sisto, Contracting elements and random walks, arXiv:1112.2666v1.

[ST89] P. Scott and T. Tucker, Some examples of exotic noncompact 3-manifolds, Quart. J. Math. Oxford Ser. (2) 40 (1989), no. 160, 481-499.

[SY94] R. Schoen and S.-T. Yau, Lectures on differential geometry, Conference Proceedings and Lecture Notes in Geometry and Topology, I, International Press, Cambridge, MA, 1994.

[Thu] W. P. Thurston, The geometry and topology of three-manifolds, Princeton lecture notes (1978-1981).

[Tuk94] P. Tukia, Convergence groups and Gromov's metric hyperbolic spaces, New Zealand J. Math. 23 (1994), no. 2, 157-187.

[WL97] M.-T. Wang and C.-H. Lin, A note on the exhaustion function for complete manifolds, Tsing Hua lectures on geometry \& analysis (Hsinchu, 1990-1991), Int. Press, Cambridge, MA, 1997, pp. 269-277.

[Wu] Y. Wu, Translation lengths of parabolic isometries of CAT(0) spaces and their applications, http://math.rice.edu/ yw22/.

[Yoo04] S. Y. Yoon, A lower bound to the action dimension of a group, Algebr. Geom. Topol. 4 (2004), 273-296 (electronic).

[Zhe96] F. Zheng, Examples of non-positively curved Kähler manifolds, Comm. Anal. Geom. 4 (1996), no. 1-2, 129-160.

Igor Belegradek, School of Mathematics, Georgia Institute of Technology, AtLANTA, GA 30332-0160

E-mail address: ib@math.gatech.edu 